\title{
Genome wide expression analysis of CBS domain containing proteins in Arabidopsis thaliana (L.) Heynh and Oryza sativa L. reveals their developmental and stress regulation Hemant R Kushwaha1,3, Anil K Singh², Sudhir K Sopory², Sneh L Singla- Pareek $^{2}$ and Ashwani Pareek*3
}

Address: ${ }^{1}$ Centre for Computational Biology and Bioinformatics, School of Information Technology, Jawaharlal Nehru University, New Delhi 110067, India, 2Plant Molecular Biology, International Centre for Genetic Engineering and Biotechnology, New Delhi 110067, India and ${ }^{3}$ Stress Physiology and Molecular Biology, School of Life Sciences, Jawaharlal Nehru University, New Delhi 110067, India

Email: Hemant R Kushwaha - ritturajhemant@gmail.com; Anil K Singh - anils13@gmail.com; Sudhir K Sopory - sopory@icgeb.res.in; Sneh L Singla-Pareek - sneh@icgeb.res.in; Ashwani Pareek* - ashwanip@mail.jnu.ac.in

* Corresponding author

Published: 28 April 2009

BMC Genomics 2009, 10:200 doi:10.1 186/147|-2164-10-200
Received: 2 December 2008

Accepted: 28 April 2009

This article is available from: http://www.biomedcentral.com/I47I-2/64/10/200

(c) 2009 Kushwaha et al; licensee BioMed Central Ltd.

This is an Open Access article distributed under the terms of the Creative Commons Attribution License (http://creativecommons.org/licenses/by/2.0), which permits unrestricted use, distribution, and reproduction in any medium, provided the original work is properly cited.

\begin{abstract}
Background: In Arabidopsis thaliana (L.) Heynh and Oryza sativa L., a large number of genes encode proteins of unknown functions, whose characterization still remains one of the major challenges. With an aim to characterize these unknown proteins having defined features (PDFs) in plants, we have chosen to work on proteins having a cystathionine $\beta$-synthase (CBS) domain. CBS domain as such has no defined function(s) but plays a regulatory role for many enzymes and thus helps in maintaining the intracellular redox balance. Its function as sensor of cellular energy has also been widely suggested.

Results: Our analysis has identified 34 CBS domain containing proteins (CDCPs) in Arabidopsis and 59 in Oryza. In most of these proteins, CBS domain coexists with other functional domain(s), which may indicate towards their probable functions. In order to investigate the role(s) of these CDCPs, we have carried out their detailed analysis in whole genomes of Arabidopsis and Oryza, including their classification, nomenclature, sequence analysis, domain analysis, chromosomal locations, phylogenetic relationships and their expression patterns using public databases (MPSS database and microarray data). We have found that the transcript levels of some members of this family are altered in response to various stresses such as salinity, drought, cold, high temperature, UV, wounding and genotoxic stress, in both root and shoot tissues. This data would be helpful in exploring the so far obscure functions of CBS domain and CBS domain-containing proteins in plant stress responses.
\end{abstract}

Conclusion: We have identified, classified and suggested the nomenclature of CDCPs in Arabidopsis and Oryza. A comprehensive analysis of expression patterns for CDCPs using the already existing transcriptome profiles and MPSS database reveals that a few CDCPs may have an important role in stress response/tolerance and development in plants, which needs to be validated further through functional genomics. 


\section{Background}

All eukaryotic genomes sequenced so far, contain a number of genes that encode for proteins whose functions are still unknown. These proteins have been documented to be induced under specific set of conditions and participate in protein-protein interactions and/or sometimes are also associated with mutant phenotypes [1]. These proteins with unknown functions are either called proteins with obscure features (POFs) when they contain no previously defined domains/motifs, or proteins with defined features (PDFs) when they contain at least one previously defined domain/motif $[1,2]$. A protein domain is an evolutionarily conserved unit of protein sequence that can evolve, function and exist independently of the rest of the protein chain. In general, each domain is assumed to perform a specific function. An identical domain may appear in evolutionarily and functionally unrelated proteins, and therefore it is challenging to relate the presence of a domain with overall functionality of the protein. One of the possible approaches to address this important issue is to use the microarray data as a tool to predict the function of proteins having unknown functions, as suggested [3]. Recently, a number of these kinds of proteins have been characterized in Arabidopsis and Oryza using transcriptome studies as well as functional genomics tools, by raising transgenic plants. It has been reported that some of these proteins of unknown function(s) can indeed improve tolerance of transgenic plants to oxidative stress [4].

To understand the probable mechanism of abiotic stress tolerance in Oryza sativa, we have made an attempt to characterize several unknown members of the stress responsive machinery [5]. A group of these proteins of unknown functions were found to have cystathionine- $\beta$ synthase (CBS) domain and were differentially regulated in the contrasting genotypes of rice indicating towards their probable role in salinity tolerance. Thus, we assume that these proteins may be participating in known pathways and networks and/or be involved in basic or specialized processes and also might comprise new and undiscovered pathways.

CBS domains are found to be associated with several proteins of unrelated functions, such as inosine-5'-monophosphate dehydrogenase (IMPDH), AMP-activated protein kinase (AMPK), chloride channels (CLC) and cystathionine- $\beta$-synthase (CBS). The importance of CBS domain was realized by the observation that point mutations in the CBS domain cause several hereditary diseases in humans [6]. CBS domain was first discovered by Bateman [7] in the genome of the archaebacterium Methanococcus jannaschii as a conserved domain in a group of proteins. CBS domain exists not only in archaebacterial proteins, but also in eubacterial and eukaryotic proteins [6]. The name of the CBS domain was coined after its dis- covery in human CBS enzyme, which is the first enzyme involved in the reverse transsulfuration pathway in which homocysteine is converted to cysteine via cystathionine. In plants and bacteria, transsulfuration pathway operates in forward direction leading to conversion of cysteine to homocysteine by the action of cystathionine- $\gamma$-synthase and $\beta$-cystathionase. While in mammals, reverse transsulfuration pathway is found in which cysteine is derived from homocysteine by CBS and $\gamma$-cystathionase enzymes (Figure 1). Yeast and some archaebacteria possess both transsulfuration pathways [8].

In CBS protein, C-terminal CBS domain exerts an autoinhibitory effect on the CBS activity, while binding of SAM (S-adenosylmethionine) with CBS domain induces a conformational change which relieves the autoinhibitory effect. Mutation in CBS domains abolish or strongly reduce activation by SAM and cause homocystinuria. CBS domain of $\gamma$-subunit of AMPK acts as sensor of cellular energy status and mutations cause a glycogen storage disease, which is clinically expressed as a familial hypertrophic cardiomyopathy (Wolff-Parkinson-White syndrome) [9-12]. Scott et al. [13] reported that CBS domain of IMPDH binds to ATP in a positive cooperative way and activates IMPDH. ATP binding and activation was abolished by a point mutation which corresponds to the mutation causing retinitis pigmentosa [14]. Earlier research shows that the CBS module in ATP-binding cassette transporter OpuA constitutes the ionic strength sensor whose activity is modulated by the C-terminal anionic tail [15]. In CLCs, function of CBS domain remains unre-

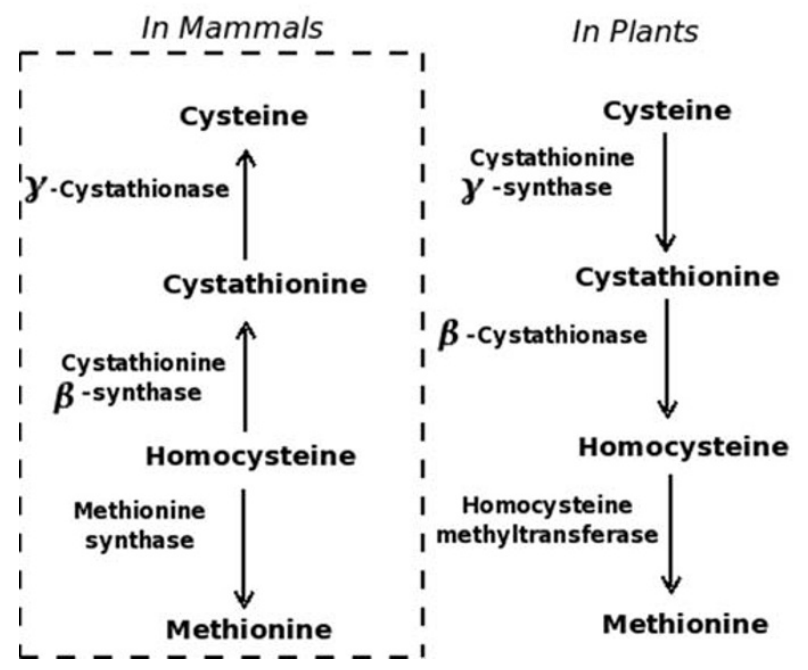

Figure I

Comparison of transulfuration pathway in plants and mammals showing role of Cystathionine $\beta$-synthase enzyme. 
solved and controversial. However, it has been shown that CBS domains in human CLCs are required for its function and/or expression because mutations in the CBS domain of CLCs cause diseases due to CLC dysfunction [16-21]. However, in plants information available on CLCs is very limited. The first CLC gene (CLC-Nt1) was identified from tobacco [22], thereafter CLC genes were identified and characterized from Arabidopsis [23-25] and rice [26], but function of CBS domain in these CLC channel genes has not been resolved.

The availability of complete genome sequence along with the microarray expression and Massively Parallel Signature Sequencing (MPSS) data makes Arabidopsis an ideal plant for study of newly identified protein family [27]. In the present work, we have performed genome wide analysis in the two highly finished plant genomes i.e. Arabidopsis thaliana and Oryza sativa where we have identified and classified the CDCPs based on their conserved features. To further establish their possible involvement in development and abiotic stresses, we have analyzed the expression of genes encoding CDCPs using MPSS database and already existing Arabidopsis microarray database http://www.arabidopsis.org.

\section{Results and Discussion Identification and classification of CBS domain containing proteins}

In the present study, we provide information on the CDCPs in Arabidopsis thaliana and Oryza sativa. For this, the CDCPs were searched against the protein sequence database for Arabidopsis and Oryza, using the HMM profile of CBS domain obtained from Pfam database as described earlier [28]. We propose a systematic classification of all the CDCPs based on their structural features, as there are no pre-existing reports on classification of CDCPs, till date.

Whole genome analysis of Arabidopsis and Oryza, employing the standard bioinformatics tools (described in methods), identified a total of 34 proteins (encoded by 33 genes) in Arabidopsis and 59 proteins (encoded by 37 genes) in Oryza with a distinct CBS domain (Table 1). These proteins could be classified into two major groups comprising of proteins containing only a single and those with two CBS domains. Apart from CBS domain, some of these proteins also possess other structural domains based on which we have further classified these proteins into subgroups. Single CBS domain containing proteins were further classified into 6 subgroups in Arabidopsis, while in Oryza CDCPs were classified into 7 subgroups. Two CBS domain containing proteins were classified into 2 subgroups in both, Arabidopsis and Oryza (Figure 2). The other observed structural domains were: CorC_HlyC $[29,30]$, voltage chloride channel (CLC) [31-33], sugar isomerase
(SIS) [34,35], pentatricopeptide repeats (PPR) [36-38], Phox and Bem1p (PB1) [39-41] and inosine monophosphate dehydrogenase (IMPDH) [14]. Some of the members of the CBS domains containing proteins also possess domain of unknown function (DUF21), while few other proteins only possess the CBS domain(s) in their sequences. In Arabidopsis, 25 proteins containing a single CBS domain were found which are encoded by 24 genes since one gene encoded for two alternate splice variants, whereas in Oryza 46 proteins containing a single CBS domain were found to be encoded by 37 genes. Interestingly, in Oryza an additional class of CDCPs having IMPDH domain was observed, which was not found in Arabidopsis. The existence of more number of CDCPs in Oryza is due to alternative splice events which correspond to the earlier report where it has been shown that in Oryza 36,650 alternate splicing events affected 8,772 genes, while in Arabidopsis 16,252 alternate splicing events affected 5,313 genes [42]. In case of two CBS domain containing proteins, 9 genes encode for 9 proteins in Arabidopsis, as no case of alternative splicing was observed, whereas in Oryza 8 genes encode for 13 proteins. The nomenclature has been assigned according to the domain(s) present in the given sequence such as CBSX for proteins containing only a single CBS domain, CBSDUFCH1 for protein containing one CBS domain along with DUF and CorC_HlyC domains, CBSCLC for proteins containing one CBS domain and a CLC domain, CBSDUF for proteins containing one CBS domain and a DUF domain, CBSSIS for proteins containing one CBS domain and a SIS domain and CBSPPR for proteins containing one CBS and a PPR domain CBSIMPDH for proteins containing one CBS domain along with an IMPDH domain. Whereas, for two CBS domain containing proteins, CBSCBS nomenclature was given to the proteins containing only two CBS domains, and CBSCBSPB for proteins containing two CBS domains and one $\mathrm{PB} 1$ domain (Figure 2). A prefix At in case of A. thaliana and Os in case of O. sativa proteins was assigned. For convenience, the alternative spliced forms were named as the gene name followed by postscript alphabets such as 'a', 'b' and so on.

\section{Analysis of CBS domain containing proteins}

Whole genome analysis of CDCPs in Arabidopsis reveals the presence of 24 genes which code for 25 proteins containing only single CBS domain, while in Oryza 29 genes coding for 46 proteins containing only single CBS domain were found. In case of two CBS domain proteins, 9 genes code for 9 proteins in Arabidopsis, while in Oryza 8 genes code for 13 proteins [Table 1]. These proteins were further classified on the basis of additional domain(s) present within the sequence. The different sub-classes of these proteins are as follows: 
Table I: CBS domain containing proteins in Oryza sativa and Arabidopsis thaliana. In O. sativa, the genes encoding CBS domain proteins were named according to their classification prefixed by 'Os' while in $A$. thailana genes were prefixed by 'At'. The alternative spliced forms were post fixed with the alphabets like 'a', 'b' and so on.

\begin{tabular}{|c|c|c|c|c|c|}
\hline Gene & Proteins & TIGR id & Locus & Coordinate & AA \\
\hline \multicolumn{6}{|c|}{ Oryza sativa sub japonica one CBS domain containing proteins } \\
\hline OsCBSXI & OsCBSXI & 12008.m06246 & LOC_Os08g22149.1 & $13226547-13234580$ & 235 \\
\hline OsCBSX2 & OsCBSX2 & I2009.m03643 & LOC_Os09g027I0.1 & $1223593-1220510$ & 227 \\
\hline \multirow[t]{5}{*}{ OsCBSX3 } & OsCBSX3a & I2002.m 10750 & LOC_Os02g57280.1 & $35086529-35087614$ & 212 \\
\hline & OsCBSX3b & I2002.m33940 & LOC_Os02g57280.4 & $35086529-35087614$ & 199 \\
\hline & OsCBSX3c & I2002.m33942 & LOC_Os02g57280.5 & $35086529-35087614$ & 197 \\
\hline & OsCBSX3d & |2002.m3394| & LOC_Os02g57280.6 & $35086529-35087614$ & 190 \\
\hline & OsCBSX3e & I2002.m33943 & LOC_Os02g57280.7 & $35086529-35087614$ & 190 \\
\hline \multirow[t]{6}{*}{ OsCBSX4 } & OsCBSX4a & |2003.m I0243 & LOC_Os03g52690.I & $30158802-30157593$ & 230 \\
\hline & OsCBSX4b & I2003.m34922 & LOC_Os03g52690.2 & $30158802-30157394$ & 205 \\
\hline & OsCBSX4c & I2003.m34923 & LOC_Os03g52690.3 & $30158802-30157394$ & 205 \\
\hline & OsCBSX4d & I2003.m34924 & LOC_Os03g52690.4 & $30158802-30157394$ & 205 \\
\hline & OsCBSX4e & |2003.m I0|489 & LOC_Os03g52690.5 & $30158802-30157593$ & 170 \\
\hline & OsCBSX4f & I3103.m05744 & LOC_Os03g52690.8 & $30203267-30199873$ & 231 \\
\hline OsCBSX5 & OsCBSX5 & I2004.m05803 & LOC_Os04g050I0.I & $24|6552-24| 4325$ & 220 \\
\hline OsCBSX6 & OsCBSX6 & |200|.m 0692 & LOC_Os0Ig44360.I & $25775244-2577736$ I & 258 \\
\hline \multirow[t]{2}{*}{ OsCBSX7 } & OsCBSX7a & $|200| . m|03| 4$ & LOC_Os0Ig40420.2 & $23|4828|-23 \mid 47344$ & 228 \\
\hline & OsCBSX7b & |200|.m97525 & LOC_Os0Ig40420.3 & $23|4828|-23 \mid 47344$ & 228 \\
\hline OsCBSX8 & OsCBSX8 & |2003.m I| 263 & LOC_Os03g63940.I & $36063946-36070376$ & 493 \\
\hline OsCBSX9 & OsCBSX9 & I2002.m05989 & LOC_Os02g064I0.I & $3204142-3206178$ & 421 \\
\hline OsCBSXIO & OsCBSXIO & |200|.m 0682 & LOC_Os0Ig44250.I & $25695370-25697808$ & 407 \\
\hline OsCBSXII & OsCBSXII & I2002.m09299 & LOC_Os02g42640.I & $25640848-25642222$ & 417 \\
\hline OsCBSXI2 & OsCBSXI2 & |2004.m I07|3 & LOC_Os04g583I 0.1 & $34488888-34487614$ & 398 \\
\hline OsCBSCLCI & OsCBSCLCI & |200|.m |266| & LOC_Os0Ig65500.2 & $38344981-38348540$ & 784 \\
\hline OsCBSCLC2 & OsCBSCLC2 & |200|.m | 267 & LOC_Os0Ig50860.I & $29546209-29548214$ & 424 \\
\hline \multirow[t]{2}{*}{ OsCBSCLC3 } & OsCBSCLC3a & I2002.m08607 & LOC_Os02g35190.1 & $21150763-21145109$ & 804 \\
\hline & OsCBSCLC3b & 12002.m33720 & LOC_Os02g35190.2 & $21150763-21145109$ & 804 \\
\hline
\end{tabular}


Table I: CBS domain containing proteins in Oryza sativa and Arabidopsis thaliana. In O. sativa, the genes encoding CBS domain proteins were named according to their classification prefixed by 'Os' while in A. thailana genes were prefixed by 'At'. The alternative spliced forms were post fixed with the alphabets like 'a', 'b' and so on. (Continued)

\begin{tabular}{|c|c|c|c|c|c|}
\hline OsCBSCLC4 & OsCBSCLC4 & I2003.m09895 & LOC_Os03g48940.3 & $27815829-278 \mid 1366$ & 508 \\
\hline OsCBSCLC5 & OsCBSCLC5 & |2004.m l04|3 & LOC_Os04g55210.I & $32601607-32606015$ & 808 \\
\hline \multirow[t]{2}{*}{ OsCBSCLC6 } & OsCBSCLC6a & $12008 . \mathrm{m} 06 \mid 20$ & LOC_Os08g20570.I & $123444 \mid 8-12350373$ & 796 \\
\hline & OsCBSCLC6b & $13108 . \mathrm{m} 09367$ & LOC_Os08g20570.2 & $12349765-12355720$ & 797 \\
\hline OsCBSCLC7 & OsCBSCLC7 & I20I2.m06347 & LOC_Os I 2g25200.I & | $4487588-14485027$ & 625 \\
\hline OsCBSCLC8 & OsCBSCLC8 & |2008.m0788| & LOC_Os08g38980.I & $24506315-245 \mid 2370$ & 750 \\
\hline \multirow[t]{2}{*}{ OsCBSCLC9 } & OsCBSCLC9a & I2002.m09922 & LOC_Os02g48880.I & $29898156-29893927$ & 783 \\
\hline & OsCBSCLC9b & |2002.m3384| & LOC_Os02g48880.2 & $29898156-29894593$ & 693 \\
\hline \multirow[t]{2}{*}{ OsCBSCLCIO } & OsCBSCLCIOa & |3104.mI| 737 & LOC_Os04g36560.I & $21892144-21886597$ & 800 \\
\hline & OsCBSCLCIOb & |3104.ml|90| & LOC_Os04g36560.2 & $21892144-21886597$ & 800 \\
\hline \multirow[t]{2}{*}{ OsCBSDUFCHI } & OsCBSDUFCHIa & |2003.m0905| & LOC_Os03g39640.I & $21984229-21972448$ & 680 \\
\hline & OsCBSDUFCHIb & $13103 . \mathrm{ml} 2995$ & LOC_Os03g39640.2 & $22028109-22016078$ & 682 \\
\hline OsCBSSISI & OsCBSSISI & I 2002.m05984 & LOC_Os02g06360.I & $3179627-3182495$ & 344 \\
\hline \multirow[t]{2}{*}{ OsCBSDUFI } & OsCBSDUFIa & |2005.m075|7 & LOC_Os05g32850.I & $19149758-19156919$ & 528 \\
\hline & OsCBSDUFIb & $12005 . \mathrm{m} 278 \mid 2$ & LOC_Os05g32850.2 & $19149758-19155394$ & 507 \\
\hline OsCBSDUF2 & OsCBSDUF2 & I2003.m09725 & LOC_Os03g47I 20.1 & $26601005-26605975$ & 420 \\
\hline OsCBSDUF3 & OsCBSDUF3 & I2003.m05876 & LOC_Os03g03430.I & $|454844-| 46 \mid 230$ & 518 \\
\hline OsCBSPPRI & OsCBSPPRI & |2009.m0577| & LOC_Os09g26190.1 & I5789767 - I5799283 & 587 \\
\hline \multirow[t]{2}{*}{ OsCBSIMPDHI } & OsCBSIMPDHIa & I2003.m I0596 & LOC_Os03g56800.I & $32304776-32309729$ & 501 \\
\hline & OsCBSIMPDHIb & I2003.m34939 & LOC_Os03g56800.2 & $32304776-32308874$ & 492 \\
\hline
\end{tabular}

Arabidopsis thaliana one CBS domain containing proteins

\begin{tabular}{|c|c|c|c|c|c|}
\hline AtCBSXI & AtCBSXI & $68417 . \mathrm{m} 05232$ & At4g36910.I & $17390623-17393305$ & 236 \\
\hline$A t C B S \times 2$ & AtCBSX2 & $68417 . \mathrm{m} 04840$ & At4g34I 20.1 & $1634|094-1634365|$ & 238 \\
\hline$A t C B S \times 3$ & AtCBSX3 & $68418 . \mathrm{m} 0126 \mathrm{I}$ & At5gl0860.I & $3428892-343047$ I & 206 \\
\hline$A t C B S X 4$ & AtCBSX4 & $684 \mid 4 . \mathrm{m} 09375$ & At lg80090.I & $30 \mid 34997-30136833$ & 402 \\
\hline$A t C B S \times 5$ & AtCBSX5 & $68417 . \mathrm{m} 03946$ & At4g27460.I & $137329 \mid 6-13734310$ & 391 \\
\hline$A t C B S \times 6$ & AtCBSX 6 & 684 I4.m07407 & At $\lg 65320.1$ & $24260925-24262136$ & 425 \\
\hline AtCBSDUFCHI & AtCBSDUFCHI & $68416 . \mathrm{m} 01634$ & At3g|3070.I & $4191518-4195119$ & 661 \\
\hline AtCBSDUFCH2 & AtCBSDUFCH2 & $684|4 . \mathrm{m} 064| 5$ & At $\lg 55930.1$ & 20922385-2092589| & 653 \\
\hline
\end{tabular}


Table I: CBS domain containing proteins in Oryza sativa and Arabidopsis thaliana. In O. sativa, the genes encoding CBS domain proteins were named according to their classification prefixed by 'Os' while in A. thailana genes were prefixed by 'At'. The alternative spliced forms were post fixed with the alphabets like 'a', 'b' and so on. (Continued)

\begin{tabular}{|c|c|c|c|c|c|}
\hline \multirow[t]{2}{*}{ AtCBSCLCI } & AtCBSCLCla & $684 \mid 4 . \mathrm{m} 06366$ & Atlg55620.I & $20790872-20794855$ & 585 \\
\hline & AtCBSCLCIb & $684 \mid 4 . \mathrm{m} 06367$ & Atlg55620.2 & $20790872-20794855$ & 781 \\
\hline AtCBSCLC2 & AtCBSCLC2 & $68418 . \mathrm{m} 03129$ & At5g26240.I & $9189527-9194734$ & 792 \\
\hline AtCBSCLC3 & AtCBSCLC3 & $68417 . \mathrm{m} 05035$ & At4g35440.I & $|683599|-\mid 6839380$ & 710 \\
\hline AtCBSCLC4 & AtCBSCLC4 & $68418 . \mathrm{m} 06178$ & At5g49890.I & $20305346-20309576$ & 779 \\
\hline AtCBSCLC5 & AtCBSCLC5 & $68418 . \mathrm{m} 04965$ & At5g40890.I & $16398542-16402547$ & 775 \\
\hline AtCBSCLC6 & AtCBSCLC6 & $68418 . \mathrm{m} 03944$ & At5g33280.I & $125665 \mid 0-12569737$ & 763 \\
\hline AtCBSCLC7 & AtCBSCLC7 & $68416 . \mathrm{m} 03398$ & At3g27I70.I & $10025254-10028435$ & 780 \\
\hline AtCBSDUFI & AtCBSDUFI & $68417 . \mathrm{m} 02197$ & At4g|4240.I & $8204342-8207340$ & 494 \\
\hline AtCBSDUF2 & AtCBSDUF2 & $68417 . \mathrm{m} 02196$ & At4gl4230.I & $8200707-8203215$ & 495 \\
\hline AtCBSDUF3 & AtCBSDUF3 & $68415 . \mathrm{m01} 625$ & At2gl4520.I & $6189275-6191730$ & 423 \\
\hline AtCBSDUF4 & AtCBSDUF4 & $684 I 4 . m 00305$ & At lg03270.I & $799191-802436$ & 499 \\
\hline AtCBSDUF5 & AtCBSDUF5 & $68418 . \mathrm{m} 0655 \mathrm{I}$ & At5g52790.I & $2|408943-2| 4 \mid 1585$ & 500 \\
\hline AtCBSDUF6 & AtCBSDUF6 & $68417 . \mathrm{m} 04786$ & At4g33700.I & $16176403-16179482$ & 424 \\
\hline AtCBSDUF7 & AtCBSDUF7 & $684 \mid 4 . m 05240$ & At $\lg 47330.1$ & $17353490-17356733$ & 527 \\
\hline AtCBSSISI & AtCBSSISI & $68416 . \mathrm{m} 0605 \mathrm{I}$ & At3g54690.I & $20257517-20259002$ & 350 \\
\hline AtCBSPPRI & AtCBSPPRI & $68418 . \mathrm{m01} 237$ & At5gl0690.I & 3374394-3377431 & 580 \\
\hline \multicolumn{6}{|c|}{ Oryza sativa sub japonica two CBS domain containing proteins } \\
\hline OsCBSX7 & OsCBSCBSI & |200|.m97524 & LOC_Os0Ig40420.I & $23|4828|-23 \mid 47344$ & 450 \\
\hline OsCBSCBS2 & OsCBSCBS2 & |200|.m|3024 & LOC_Os0Ig69240.I & $40567292-40565692$ & 435 \\
\hline OsCBSCBS3 & OsCBSCBS3 & I2004.m08236 & LOC_Os04g31340.I & $18564463-18559754$ & 425 \\
\hline \multirow[t]{5}{*}{ OsCBSCBS4 } & OsCBSCBS4a & I2004.m35295 & LOC_Os04g32880.I & $19671270-19664088$ & 451 \\
\hline & OsCBSCBS4b & I2004.m I0|520 & LOC_Os04g32880.2 & $19671270-19664088$ & 451 \\
\hline & OsCBSCBS4c & I2004.m08385 & LOC_Os04g32880.4 & $19668390-19664088$ & 346 \\
\hline & OsCBSCBS4d & I2004.m35296 & LOC_Os04g32880.3 & $19671270-19665094$ & 405 \\
\hline & OsCBSCBS4e & |3104.m I|890 & LOC_Os04g32880.5 & $19686702-19679520$ & 452 \\
\hline OsCBSCBS5 & OsCBSCBS5 & $|200| . m \mid 3010$ & LOC_Os0lg69090.I & $40484031-40480347$ & 404 \\
\hline OsCBSCBSPBI & OsCBSCBSPBI & |200|.m|3039 & LOC_Os0Ig69900.I & $407|2332-407| 5773$ & 552 \\
\hline
\end{tabular}


Table I: CBS domain containing proteins in Oryza sativa and Arabidopsis thaliana. In O. sativa, the genes encoding CBS domain proteins were named according to their classification prefixed by 'Os' while in A. thailana genes were prefixed by 'At'. The alternative spliced forms were post fixed with the alphabets like 'a', 'b' and so on. (Continued)

\begin{tabular}{llccc}
\hline OsCBSCBSPB2 & OsCBSCBSPB2 & I20II.m04890 & LOC_OsIIg06930.I & $3419765-3414996$ \\
\hline OsCBSCBSPB3 & OsCBSCBSPB3 & I200I.mI3334 & LOC_Os0Ig73040.I & $42704156-42708054$ \\
\hline OsCBSCBSPB4 & OsCBSCBSPB4 & I20I2.m04708 & LOC_OsI2g07I90.I & $3535282-35307 \mid 8$ \\
\hline
\end{tabular}

Arabidopsis thaliana two CBS domain containing proteins

\begin{tabular}{|c|c|c|c|c|c|}
\hline$A t C B S C B S P B I$ & AtCBSCBSPBI & $68418 . \mathrm{m} 07970$ & At5g63490.I & $25435820-25439410$ & 543 \\
\hline AtCBSCBSPB2 & AtCBSCBSPB2 & $68415 . \mathrm{m} 04480$ & At2g36500.I & $|5325| 07-|532723|$ & 536 \\
\hline AtCBSCBSPB3 & AtCBSCBSPB3 & $68416 . \mathrm{m} 05837$ & At3g52950.I & $19645474-19647797$ & 556 \\
\hline AtCBSCBSPB4 & AtCBSCBSPB4 & $68418 . \mathrm{m} 06258$ & At5g50530.I & $20589102-20592159$ & 548 \\
\hline AtCBSCBSPB5 & AtCBSCBSPB5 & $68418 . \mathrm{m} 06274$ & At5g50640.I & $20622444-20625490$ & 548 \\
\hline AtCBSCBSI & AtCBSCBSI & $68416 . \mathrm{m} 05299$ & At3g48530.I & $179984 \mid 7-18000748$ & 424 \\
\hline$A t C B S C B S 2$ & AtCBSCBS2 & $684|4 . \mathrm{m} 0803|$ & Atlg69800.1 & $26277893-26279992$ & 447 \\
\hline AtCBSCBS3 & AtCBSCBS3 & $68414 . \mathrm{m} 01006$ & Atlg09020.I & $2899918-2904818$ & 487 \\
\hline AtCBSCBS4 & AtCBSCBS4 & $684 \mid 4 . \mathrm{m} 01834$ & Atg $\lg 15330.1$ & $527434 \mid-5275597$ & 352 \\
\hline
\end{tabular}

\section{A. CBSX}

A fraction of predicted CBS domain-containing proteins harbor only single CBS domain (PF00571). CDCPs have been reported to have the regulatory functions http:// www.sanger.ac.uk/Users/agb/CBS/CBS.html, however the biological significance of these domains remains to be elucidated. These CDCPs can act as binding domains for adenosine derivatives and may regulate the activity of attached enzymatic or other domains [43]. In some cases, these proteins may act as sensors of cellular energy status as they are activated by AMP and inhibited by ATP [44]. Recently, one of the Arabidopsis CBSX proteins (CDCP2) has been purified and crystallized [45]. In case of Arabidopsis, 6 genes were found to encode for 6 CBSX proteins, while in Oryza, a total of 22 CBSX proteins were categorized in this subgroup, which were encoded by 12 genes. In Oryza, all the CBSX genes code for only one protein, except for OsCBSX3, OsCBSX4 and OsCBSX7, which code for 5, 6 and 2 proteins, respectively through the alternate splicing mechanism.

\section{B. CBSDUFCH}

In case of Arabidopsis, this subgroup contains two proteins which are encoded by two genes, while in case of Oryza, two proteins are encoded by a single gene. These proteins contain a domain of unknown function (DUF21) (PF01595) at the N-terminus adjacent to a CBS domain and a CorC_HlyC domain (PF03471) at the C-terminus. DUF21 domain has no known function and is usually present at the $\mathrm{N}$-terminus of the proteins adjacent to a CBS domain. The CorC_HlyC is a transport associated domain and is found at the C-terminus of the proteins. CorC_HlyC domain is also found in magnesium and cobalt efflux protein CorC and some of the $\mathrm{Na}^{+} / \mathrm{H}^{+}$antiporters. The function of this domain is unknown but it might be involved in modulating the transport of ion substrates http://pfam.sanger.ac.uk.

\section{CBSDUF}

Proteins classified in this subgroup contain a DUF21 domain at the N-terminus, along with a CBS domain. In case of Oryza, this subgroup contains four proteins, which are encoded by three genes, as OsCBSDUF1 gene codes for two proteins whereas, in case of Arabidopsis, this subgroup contains seven proteins, which are encoded by seven genes.

\section{CBSCLC}

These proteins belong to chloride channel protein (CLC) family which sustains a wide variety of cellular functions, including membrane excitability, synaptic communication, transepithelial transport, cell volume recognition, cell proliferation, and acidification of endosomes and lysosomes. Earlier chimeric and deletion approaches had 


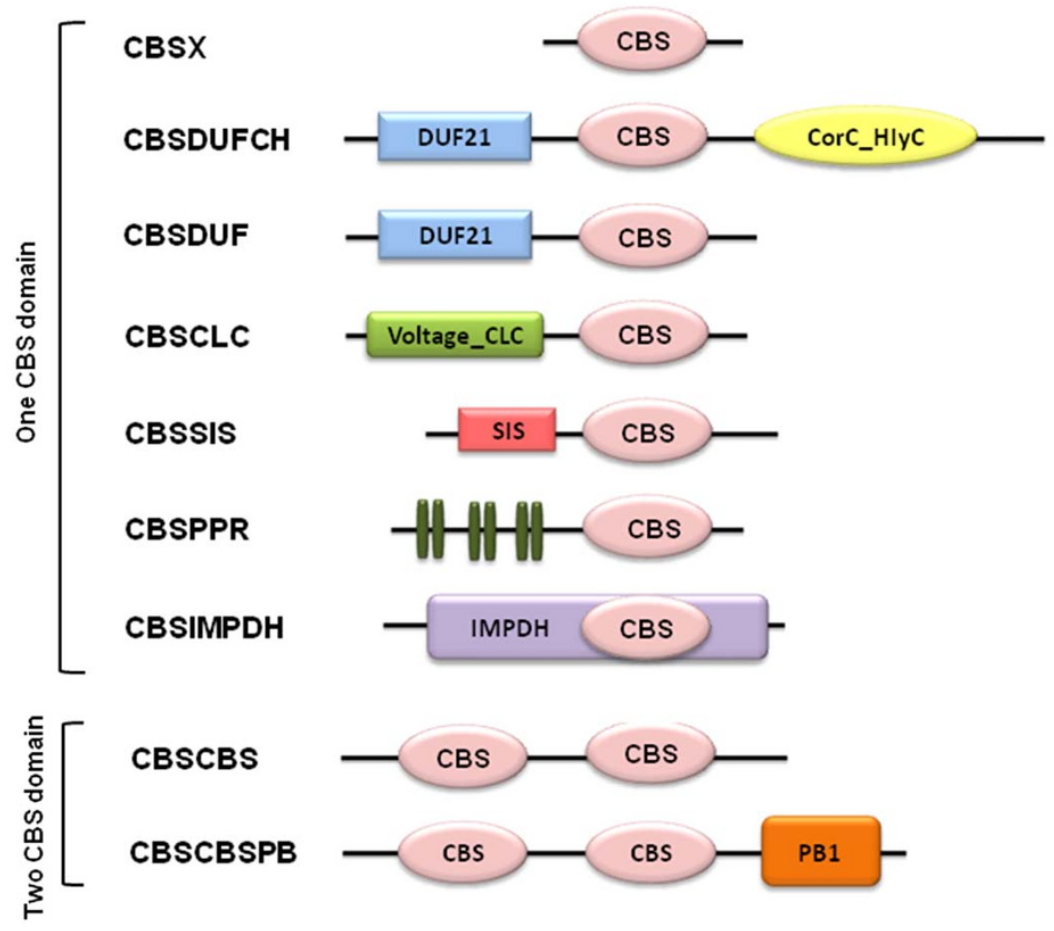

\section{Figure 2}

Representation (unscaled) of the primary domain structure of the CDCP proteins in Arabidopsis thaliana and Oryza sativa. All the CDCPs were classified into two groups; single CBS domain containing proteins and two CBS domain containing proteins. Single CBS domain containing proteins were further classified into seven subgroups based on the additional domain(s) present in their sequences. Proteins containing only single CBS domain and no other functional domain were named as CBSX. Other proteins containing single CBS domain were named according to the presence of other functional domain. Two CBS domain containing proteins were classified into two subgroups. Proteins containing only two CBS domains and no other functional domain were named as CBSCBS and proteins containing two CBS domains and one PBI domain were named as CBSCBSPB. The subfamily CBSIMPDH was observed only in $O$. sativa.

suggested that CBS domain may influence gating of CLCs. Past studies have suggested that mutation in the CBS domain affects protein-protein interaction within CLC protein subunits as well as between two subunits of the dimer and that they influence the voltage dependence of gating through the common gate [46]. Role of CBS domain in the correct targeting (possibly related to correct folding) of the CLC is in accordance with previous studies with yeast CLC protein where mutation in the CBS domain abolished its localization to the late golgi that is seen upon its overexpression [47]. Earlier experiments have shown that certain mutations in CBS domain affect chloride channel gating but physiologically relevant regulatory role of CBS domains in CLC channels is yet to be established [46]. In an earlier report, 7 CLC genes were identified each from Oryza and Arabidopsis [48]. However, in the present study we have identified total 10 genes encoding 14 CLC proteins in Oryza. Whereas in case of
Arabidopsis, our results are in accordance with the previous report as we found 8 CLC proteins encoded by 7 genes.

\section{E. CBSSIS}

Proteins containing sugar isomerase (SIS) domain along with a CBS domain have been classified in this subgroup. We have identified one CBSSIS gene encoding only one protein in cases of both, Arabidopsis and Oryza. The SIS domain is widespread and found in all species including, prokaryotic, archaebacterial and eukaryotic proteins. In general SIS domain is found in proteins that have a common role in phosphosugar isomerization as SIS domain functions by binding to the phosphosugars. The SIS domains are also found in a family of bacterial transcriptional regulators [34] as well as in a family of Escherichia coli iron transporters [49]. 


\section{F. CBSPPR}

In both Arabidopsis and Oryza, this subgroup is composed of a single gene encoding for a single protein, containing a pentatricopeptide repeat (PPR) motif and a CBS domain. The PPR motif, first described by Small et al. [36] is a degenerate 35 amino acid sequence closely related to the 34 amino acid tetratricopeptide repeats (TPR) motif. PPR repeat motifs are structural motifs encoded by a large number of genes in plants and other organisms, although the PPR gene family is greatly expanded in plants. It was hypothesized that this could be due to novel functions served by PPR proteins in plants that are not required in other organisms, or that PPR proteins replace functions performed by other genes in other organisms. Also, restoration of male fertility is a plant specific function encoded by PPR genes [50]. A genome-wide analysis of Arabidopsis PPR family proteins has identified 441 members and their further analysis revealed that PPR proteins play constitutive, often essential roles in mitochondria and chloroplast, probably via binding to organeller transcripts [51]. Many plant PPR genes that have been functionally annotated so far, are involved in either male fertility restoration through modification or silencing of cytotoxic mitochondrial transcripts, or post transcriptional modulation of plastid gene expression or plant embryogenesis and other plant developmental processes [52]. Interestingly, among large number of PPR proteins found in plants, only one PPR protein, each in Oryza and Arabidopsis, contains a CBS domain in the same reading frame. Occurrence of CBS domain along with PPR repeat in a protein suggests that this protein might be involved in the various cellular processes by sensing the cell energy status [13]. Structurally characterizing the full length protein (PPR + CBS domain) may shed light on this regulatory mechanism in plants.

\section{G. CBSIMPDH}

Proteins containing inosine-5'-monophosphate dehydrogenase (IMPDH) domain (PF00478) along with CBS domain has been classified in this subgroup. In Oryza, only one CBSIMPDH gene has been identified, which code for two CBSIMPDH proteins while in Arabidopsis, no member of this subgroup has been identified. Interestingly, in Oryza CBSIMPDH proteins, CBS domain lies within the IMPDH domain. IMPDH domain has been identified in the sequence of IMPDH enzyme, which is a key enzyme in the de novo guanosine nucleotide biosynthesis. Scott et al. [13] have shown that the CBS domain of IMPDH binds to ATP in vitro and that the tetrameric IMPDH binds ATP in a positive, cooperative way. They have also observed that IMPDH was activated by ATP, which was never reported earlier. This observation strongly supported the verity that ATP binding to the CBS domain allosterically activates IMPDH and consequently $\mathrm{XMP}$ synthesis. If so, this mechanism would couple the
GTP/dGTP biosynthesis to the cellular energy status i.e., high ATP levels [6].

\section{H. CBSCBS}

In this subgroup, proteins containing only two CBS domains have been classified. In case of Oryza, 8 CBSCBS proteins have been identified, which are encoded by 5 different genes. The OsCBSX7 gene, which has been classified in CBSX subgroup, also codes for a protein OsCBSCBS1 containing two CBS domains. The OsCBSCBS4 gene encodes for four proteins through an alternative splicing mechanism whereas, in case of Arabidopsis, AtCBSCBS subgroup contains four genes, which encode four proteins.

\section{CBSCBSPB}

These proteins contain Phox/Bemp1 (PB1) domain along with the CBS domain. In case of Oryza, this subgroup possess 4 genes, which encode for 4 proteins, while in Arabidopsis 5 CBSCBSPB genes encode for 5 proteins. The PB1 domains are present in many eukaryotic cytoplasmic signaling proteins. They are dimerization/oligomerization domains present in adaptor and scaffold proteins and kinases that serve to organize platforms that ensure specificity and fidelity during cellular signaling. Recently, a number of studies have provided valuable information on the structural details that govern binding between the different PB1 modules and explain how they direct the formation of different macromolecular signaling complexes [53]. Proteins containing the PB1 domain are conserved in animals, fungi, amoebae and plants, which participate in various biological processes [54]. The function of PB1 domain containing proteins in plants has not been reported so far. Presence of PB1 domain, along with a pair of CBS domains, in a single protein suggests that these proteins might be involved in cellular signaling processes through interaction with other proteins and/or ligands (ATP, ADP or SAM). Characterization of these proteins at physiological, molecular and structural level might shed some light on their functionality.

\section{Chromosomal distribution of CBS domain containing proteins}

In Arabidopsis, the family of 33 CDCP genes was found to be distributed randomly on all the 5 chromosomes (Figure 3a), while in Oryza the family of 37 CDCP genes was distributed on 9 out of 12 chromosomes (Figure 3b). In case of Arabidopsis, maximum (10 in number) CDCP genes, were found to be located on chromosome $\mathrm{V}$, followed by 9 on chromosome I, 7 on chromosome IV, 5 on chromosome III and 2 on chromosome II. While in Oryza maximum ( 9 in number) CDCP genes were found to be located on chromosome I, followed by 7 on chromosome III, 6 each on chromosome II and IV, 3 on chromosome 


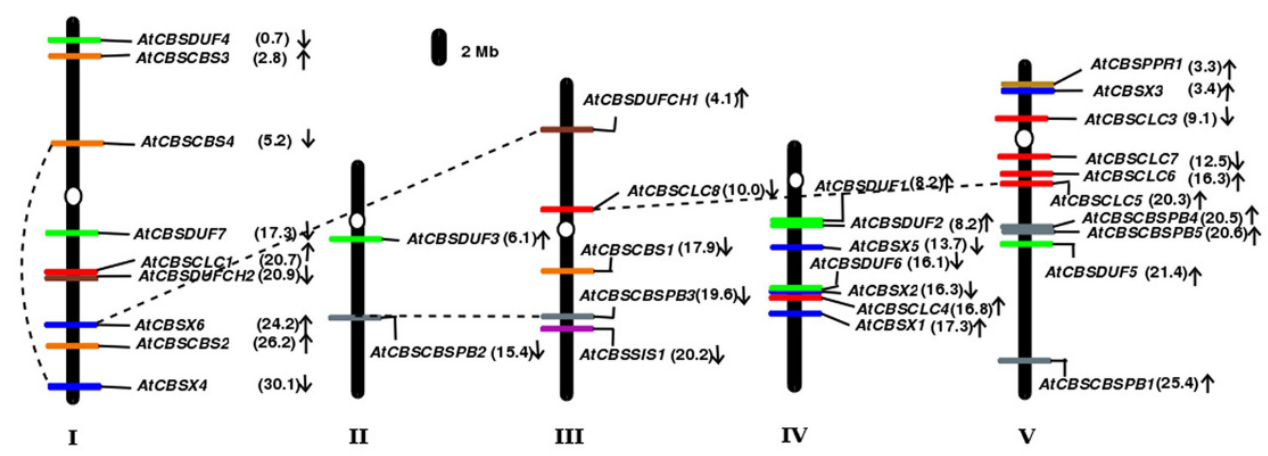

B

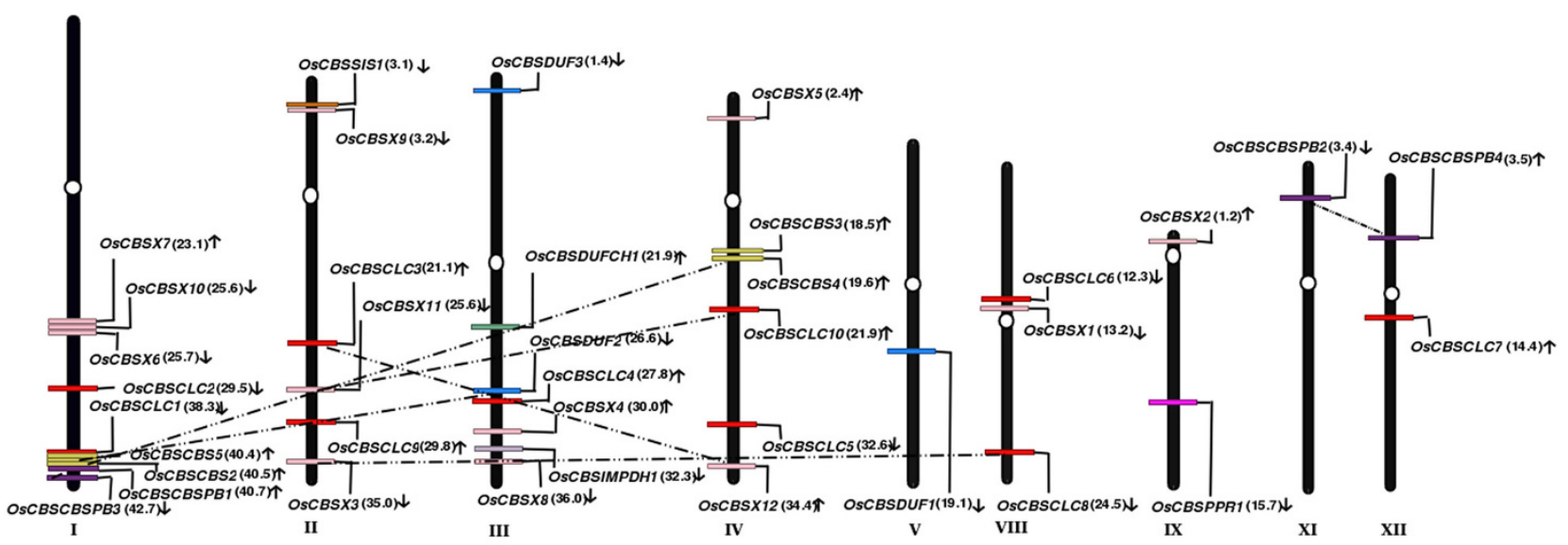

Figure 3

Genomic distribution of CDCP genes on Arabidopsis thaliana (A) and Oryza sativa (B) chromosomes. White ovals on the chromosomes (vertical bars) indicate the position of centromeres. Chromosome numbers are indicated at the bottom of each bar. The position of first exon of genes (in Mb) has been marked in the parentheses along with their names at the same location on chromosomes. Arrow marks the direction of the ORF specific to the gene encoding CDCP protein. In case of Oryza, only those chromosomes having CDCP genes are shown.

VIII, 2 each on chromosomes IX and XII; and 1 each on chromosomes $\mathrm{V}$ and VII.

The distribution of the CDCP genes on the 5 chromosome of Arabidopsis and 9 chromosome of Oryza, at which they were found to be located, is not uniform. Their chromosomal distribution pattern reveals that some CDCP genes are found in clusters on certain chromosomes at various chromosomal regions. Occurrence of cluster of genes belonging to a family at certain chromosomes and chromosomal regions is common. Jain et al. [55] have reported that among the 19 auxin responsive (SAURs) genes present on chromosome IX, 17 are clustered together at a single locus in tandem. Similarly, genes encoding basic leucine zipper transcription factors
(OsbZIP) have also been reported to be present in clusters at certain chromosomes and chromosomal regions [56]. In Oryza, 4 genes encoding two CBS domain containing proteins were found in close vicinity at chromosome I. Percent identity amongst all the four genes clustered was found to be in the range of 30 to $60 \%$.

The sequence information and analysis of the Arabidopsis [57-60] and Oryza [61] whole genomes have revealed numerous large-scale segmental duplications. Several studies conclude that at least two rounds of duplications might have probably occurred in the Arabidopsis genome, with many losses and rearrangements, leaving a mosaic of "segmental duplications" or "duplication blocks" [61-65]. Analysis of CDCP genes has revealed that some of these 
genes have been duplicated during the process of evolution in both, Arabidopsis and Oryza. Figure 3a shows the duplicated CDCP genes in Arabidopsis and Figure 3b shows the duplicated CDCP genes in Oryza. In Arabidopsis, AtCBSX4 at chromosome I seem to be duplicated segmentally, and a domain duplication event might have occurred, which have resulted in the appearance of AtCBSCBS4 gene on the same chromosome. AtCBSDUFCH 2 gene at chromosome I was observed to be duplicated to chromosome III as AtCBSDUFCH1. These duplicated genes show $85 \%$ identity at the nucleotide level and $79 \%$ identity at the protein level. AtCBSCLC8, present on the chromosome III seems to be duplicated segmentally to chromosome $\mathrm{V}$ as AtCBSCLC6 and at the same time the gene observed to have undergone inversion. AtCBSCBSPB2 present at chromosome II seems to be segmentally duplicated at chromosome III as AtCBSCBSPB3. These genes share $86 \%$ identity at the nucleotide level and $67 \%$ identity at the protein level. Interestingly in Oryza, it was observed that during the course of duplication the structural domains accumulate variations in order to adapt to new function. For example OsCBSDUF2 gene present on chromosome III got duplicated on chromosome I as OsCBSCBS5. In other instances the CDCP genes were duplicated but retained their structural domains, like OsCBSCBSPB2 present on chromosome XI got duplicated as OsCBSCBSPB4 on chromosome XII. OsCBSCLC9 on chromosome II was found to be duplicated as OsCBSCLC8 on chromosome VIII. OsCBSCBS2 located on chromosome I was observed to be duplicated as OsCBSCBS3 on chromosome IV.

\section{Sequence Analysis of CBS domain containing proteins}

Sequence analysis of all the CDCPs shows an overall homology amongst their own respective groups (see Additional files: 1 and 2). Alignment of CBS domain sequences shows that the conserved domain also possesses some variations within themselves. Single CBS domain proteins of Oryza have been observed to have a percent identity of $55 \%$ to $60 \%$ within themselves, and $30 \%$ with that of Arabidopsis except for the AtCBSX1 which showed identity of more than $80 \%$ with OsCBSX1 and OsCBSX2. Similar identity pattern was also observed for the OsCBSCLC proteins, which showed identity ranging from $30 \%$ to $80 \%$ within their own subgroups and also with AtCBSCLC proteins. The OsCBSDUF proteins were observed to have identity ranging from $50 \%$ to $60 \%$ with the AtCBSDUF proteins. The OsCBSSIS1 protein shares $80 \%$ identity with AtCBSSIS1 and OsCBSPPR1 protein shares 60\% identity with AtCBSPPR1 protein. OsCBSCBS proteins share $40 \%$ to $75 \%$ identity among themselves. The two CBS domain proteins also showed $50 \%$ to $70 \%$ identity with the other two CBS domain members. Apart from having variations, the CBS domain also accumulates large insertions. The large insertion or deletion might have helped the CBS domain proteins to evolve in order to perform specific functions. Results from the sequence alignment suggested that the sequences might have evolved according to the other functional domains present in the sequences which might have led to their specialized functions.

\section{Phylogenetic analysis of CBS domain containing proteins}

To study the phylogenetic relationship amongst the CDCPs in both Arabidopsis and Oryza, an unrooted tree was constructed from the alignment of full length protein sequences. Analysis of single CBS domain proteins in both Arabidopsis and Oryza revealed that all the single CBS domain containing proteins were divided into three clades (Figure 4). It was observed that all the OsCBSX and AtCBSX proteins clustered together in single clade, except for OsCBSX8, OsCBSX9, OsCBSX11, OsCBSX12, AtCBSX4, AtCBSX5 and AtCBSX6. The OsCBSX11 and OsCBSX12 proteins were found in the clade with CBSCLC proteins showing significant identity $(52 \%)$ with that of the CBSCLC proteins. The OsCBSSIS1 and AtCBSSIS1 proteins share cluster with majority of OsCBSX and AtCBSX proteins. It was observed that OsCBSSIS1 and AtCBSSIS1 proteins share $40 \%$ identity with each other. All the OsCBSDUF and AtCBSDUF proteins were clustered together in the same clade. AtCBSPPR1, OsCBSPPR1 and OsCBSIMPDH proteins were also found to be lying in the same clade along with CBSDUF proteins. OsCBSIMPDH proteins share $30 \%$ to $44 \%$ identity with the CBSDUF proteins. Third clade comprises of the OsCBSCLC and AtCBSCLC proteins and it was also shared by OsCBSDUFCH1 and AtCBSDUFCH1 proteins. Analysis of two CBS domain containing proteins in Arabidopsis and Oryza clearly showed three clades (Figure 5). The OsCBSCBSPB and AtCBSCBSPB proteins were observed to be clustered together in one clade, while OsCBSCBS and AtCBSCBS proteins were found to be divided in two separate clades. The first clade represented all the CBSCBS proteins except for OsCBSCBS4 and AtCBSCBS3. The large number of alternative splicing observed in OsCBSCBS4 resulted in the separate clade showing the amount of variation adopted by these proteins with respect to the other OsCBSCBS proteins. When observed at the sequence level OsCBSCBS4 was found to have $50 \%$ to $60 \%$ identity with other CBSCBS members. OsCBSCBS5 protein was found to be closer to the OsCBSCBS4 protein showing an increase in the copy number of the protein in Oryza due to evolution which might be caused in order to adapt for specific function. These results suggest that the CBS domain containing proteins might have evolved differentially in order to adapt to the specific functions. 


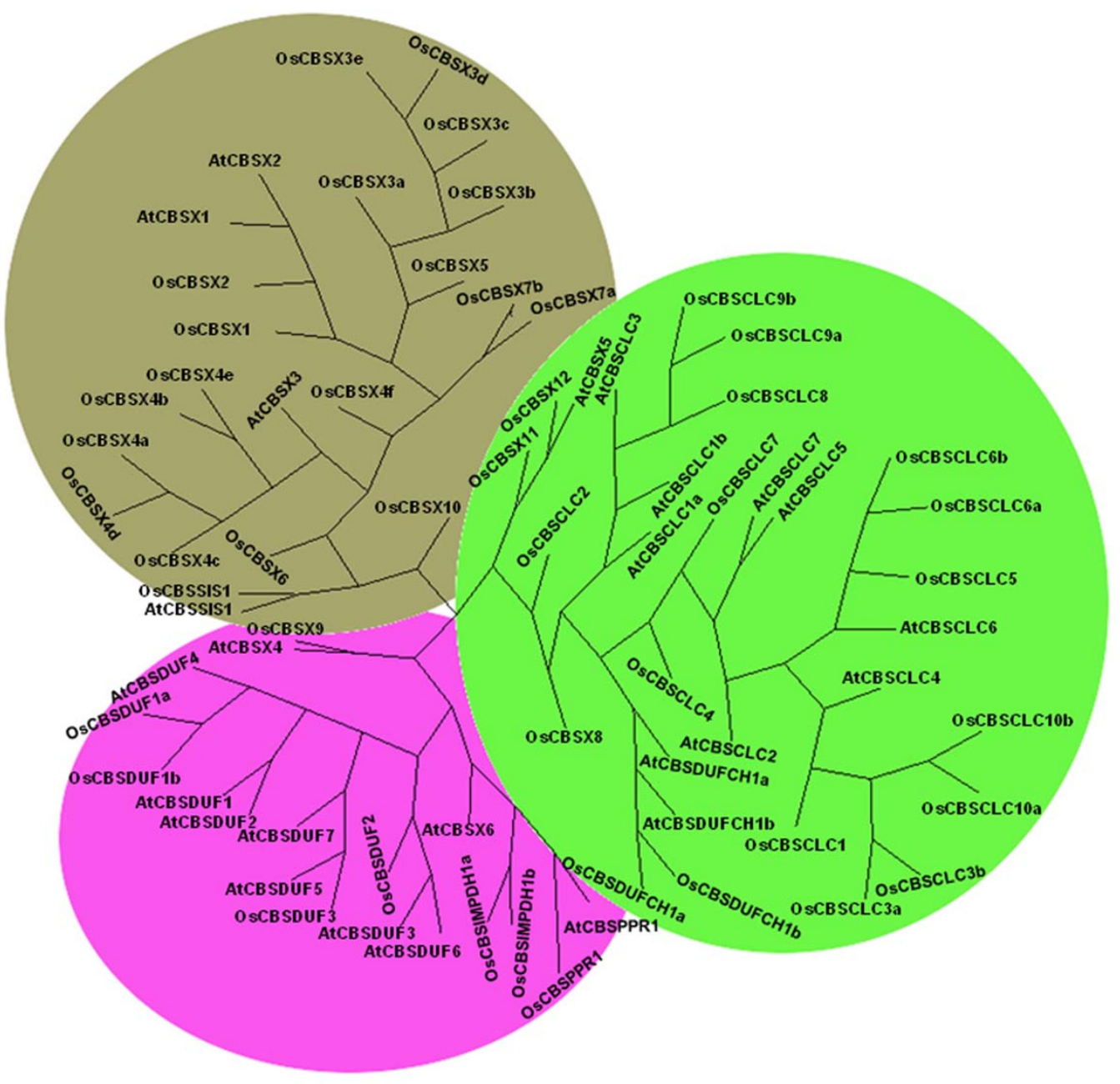

\section{Figure 4}

The unrooted parsimonious tree of single CBS domain containing proteins in A. thaliana and O. sativa showing different clusters. The tree was plotted using drawtree program of phylip software.

\section{MPSS analysis of genes encoding CBS domain containing proteins}

Massively parallel signature sequencing (MPSS) provides a sensitive quantitative measure of gene expression for nearly all genes in the genome [66]. To study the expression of CDCP genes in various tissues/organs under different conditions, we extracted the information about the MPSS tags available for both 17 base and 20 base libraries representing 6 different parts of the plant from Arabidopsis MPSS Project http://mpss.udel.edu/at/ and Oryza MPSS project http://mpss.udel.edu/rice/ (see Additional files: 3 and 4 ). The heatmaps generated from these data are presented as Figure 6 and 7.

Analysis of CDCP genes in Arabidopsis and Oryza showed that AtCBSX4 has no expression value in any of the tissues under any condition according to the MPSS database while in Oryza OsCBX12 has no expression values in the MPSS database. In Arabidopsis, genes encoding for proteins with CorC_HlyC functional domain, which has a major role in magnesium and cobalt efflux, along with CBS domain seems to be expressed more in siliqua, leaves and inflorescence. In Oryza, OsCBSDUFCH1 gene was observed to show more expression in leaves and other plant tissues, except roots. The gene AtCBSDUFCH1 is expressed more in inflorescence of ap1-10, ap3-6 mutants than in the normal condition of inflorescence. AtCBSDUFCH2, which is duplicated from AtCBSDUFCH1 on chromosome III, is expressed more in leaves and seed. In case of Arabidopsis, CBSCLC genes showed more expression in callus, roots and leaves overall in the MPSS analysis, while in case of Oryza, OsCBSCLC7 seems to be more expressed in root tissues, while other members were found to be expressed more in aerial part 


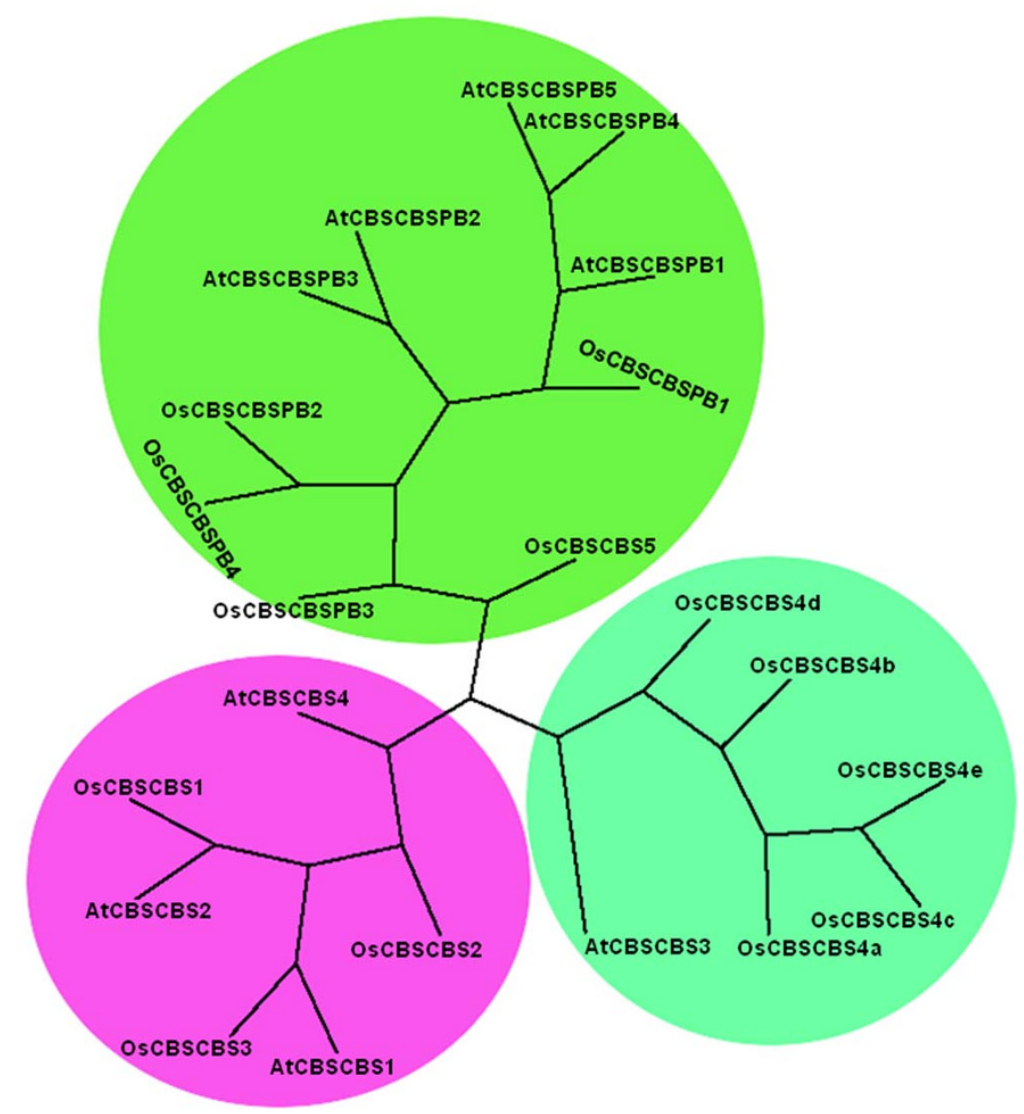

\section{Figure 5}

The unrooted parsimonious tree of two CBS domain containing proteins in $A$. thaliana and $O$. sativa showing different clusters. The tree was plotted using drawtree program of phylip software.

of the plant. It was observed that in TIGR ver 6 , the data for OsCBSCLC10 was not available in the MPSS database. The CBSSIS1 gene seems to be expressed more in callus, normal inflorescence and leaves, while CBSPPR1 gene showed more expression in leaves, seed, siliqua and roots in Arabidopsis. In Oryza CBSSIS1, CBSPPR1 and CBSIMPDH1 genes were observed to maintain a constant level of expression in all the plant tissues.

Among the groups of genes having two CBS domains, MPSS analysis showed higher expression for all the CBSCBS genes in all the plant parts in Oryza, whereas in case of Arabidopsis, AtCBSCBS4 does not show expression in any of the plant part considered for the MPSS analysis. AtCBSCBS2 showed enhanced levels of expression in normal inflorescence, leaves and callus. While, AtCBSCBS3 showed expression in other inflorescence such as ap1-10, ap3-6 and agamous mutant conditions but does not show any expression in the normal inflorescence.

In Arabidopsis, AtCBSCBSPB1, AtCBSCBSPB2 and AtCBSCBSPB3 showed expression in MPSS analysis.
Whereas in Oryza, OsCBSCBSPB3 was observed to show expression in the meristematic tissues and root while rest of the genes of this subclass were found to have been expressed in leaves also. In Arabidopsis, AtCBSCBSPB1 showed more expression in callus, root and normal inflorescence, while AtCBSCBSPB2 showed expression only in normal inflorescence and siliqua. This data indicates that CDCP genes exhibit a strict tissue specific expression which is also developmentally controlled.

\section{Expression profiles of genes encoding CBS domain containing proteins under various stresses}

To examine the expression of CBS domain containing proteins under various abiotic stress conditions in Arabidopsis, we took advantage of the available data on transcriptional profiling http://www.arabidopsis.org/. Analysis of microarray data indicated that some of the CDCP genes are regulated by various abiotic stress conditions (Figure 8 and 9).

Thomashow [67] has shown that model plant Arabidopsis may express as many as hundreds of cold shock proteins 


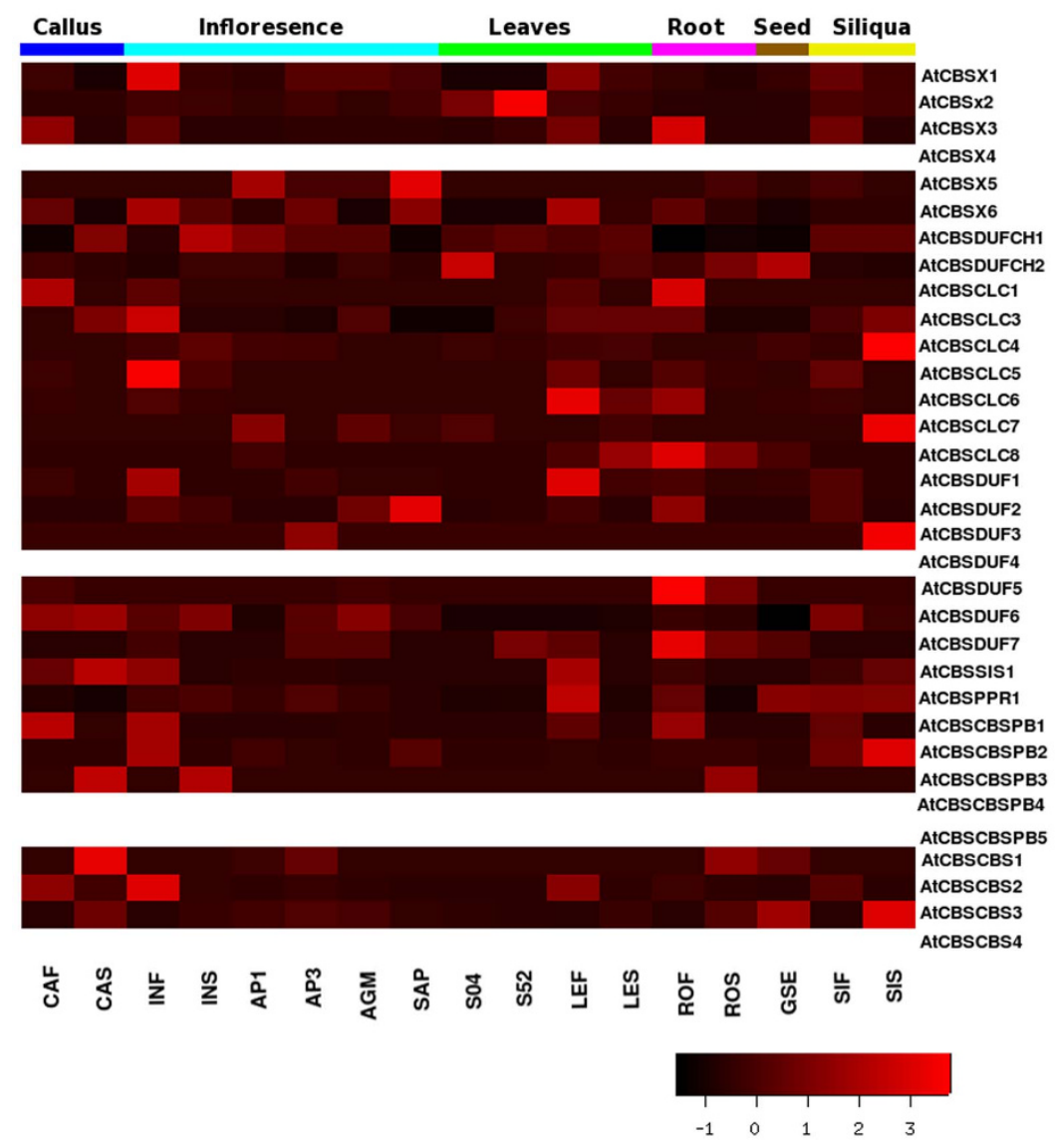

Figure 6

Heatmap of the expression analysis from the MPSS data in different tissues of the Arabidopsis thaliana. The empty rows of heatmap correspond to the absence of transcript abundance values for the respective gene. The heatmap was made using gplots package of open source R software. The scale shows the Z-score, which is defined as "actual value" minus the mean of the group divided by the standard deviation.

under cold stress condition. CDCP genes showed altered expression at $24 \mathrm{hr}$ cold stress in root with respect to the control, while in shoot some of the CDCP genes showed early upregulation within $30 \mathrm{~min}$ and $1 \mathrm{hr}$ of cold stress. Some of the CDCP genes also showed up regulation at 12 $\mathrm{hr}$ to $24 \mathrm{hr}$ cold stress conditions. AtCBSSIS1 gene was found to be upregulated in shoots under cold and wound stresses, while in roots, upregulation was observed only under osmotic and salt stresses. In roots, AtCBSDUF3 and AtCBSCLC7 showed upregulation in cold stress condition at 12 hour and 24 hour. Under cold stress condition AtCBSCBSPB4 and AtCBSCBSPB5 showed upregulation within 24 hrs in roots but in shoots they showed biphasic upregulation i.e. their expression was upregulated within 30 min of stress, followed by downregulation of expression and it was again upregulated within $24 \mathrm{hr}$ of the stress exposure. High (intense) light stress causes the formation of oxygen radicals in chloroplasts and has the potential to damage them. However, plants are able to respond to this stress and protect chloroplasts by various means, including transcriptional regulation in the nucleus. Although the corresponding signaling pathway is unknown, there have been attempts to study its regulation [68]. When exposed to UV light, CDCP genes showed upregulation at $24 \mathrm{hr}$ exposure in roots (also expressed at early timepoints), while in shoot, UV light exposure from 1 to $3 \mathrm{hrs}$ is found to be sufficient to induce the expression of CDCP genes. Most of the CDCP genes maintain a constant level of expression during all the time period of the UV exposure suggesting that these genes might play crucial role(s) in the light sensing mechanism. The complex responses of plants to wounding have been extensively studied in recent years, and numerous wound-responsive genes have been identified in Arabidopsis [69]. Wounding stress in shoot showed comparable expression at all the time points while in roots CDCP genes showed expression 


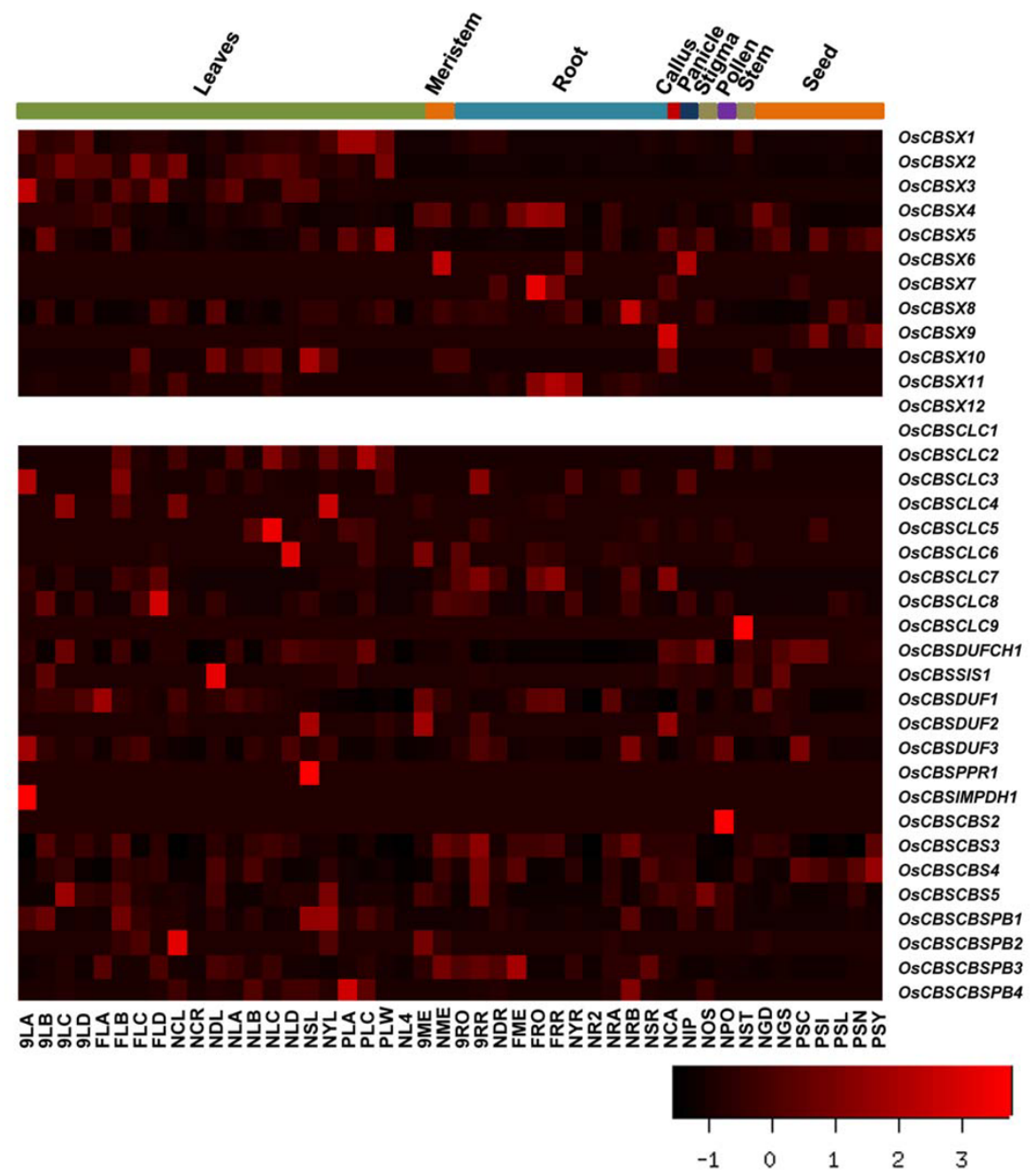

Figure 7

Heatmap of the expression analysis from the MPSS data in different tissues of the Oryza sativa. The empty rows of heatmap correspond to the absence of transcript abundance values for the respective gene. The heatmap was made using gplots package of open source R software. The scale shows the Z-score, which is defined as "actual value" minus the mean of the group divided by the standard deviation.

at $1 \mathrm{hr}$ and $24 \mathrm{hr}$ of exposure to stress. When exposed to the genotoxic stress, the cell cycle is halted to gain necessary time for repairing DNA and genes required for repair and protection of other cellular components endangered by the genotoxic stress are activated. Some organisms do respond to the abiotic stresses by way of apoptosis i.e. eliminating the damaged cell [70]. On exposure to bleomycin (genotoxic), CDCP genes showed differential expression in root and shoot at all the time points except for the $6 \mathrm{hr}$ in shoot, where none of the gene showed enhanced expression. This data indicates that the CDCPs might also play role in protecting the cells from the genotoxic stress. Under drought conditions, plants adapt themselves to maintain the cellular homeostasis. It has been observed that sensitive plants suffer rapid irreversible cell damage, essentially due to degradation of their membranes [71]. Membranes are main targets of degradative processes induced by drought and it has been shown that, under water stress, a decrease in membrane lipid content is correlated to the inhibition of lipid biosynthesis $[72,73]$ and a stimulation of lipolytic and peroxidative activities [74-77]. When observed under drought conditions, all CDCP genes showed comparable expression at different time points in root, while in shoot approximately all the CDCP genes were found to be upregulated at $24 \mathrm{hr}$ drought stress, which indicates that plant takes some time to adapt to the drought conditions, suggesting that CDCPs might also help plants to adapt to drought stress. 


\section{A}
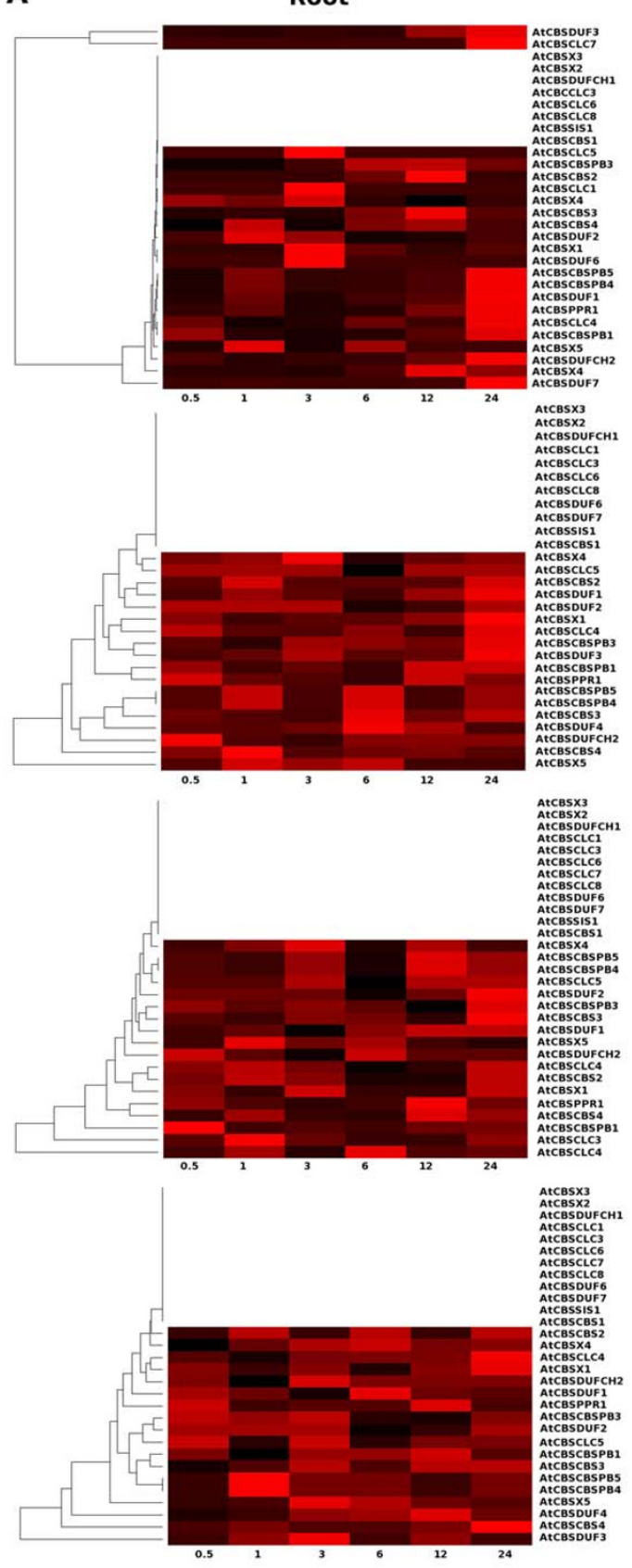
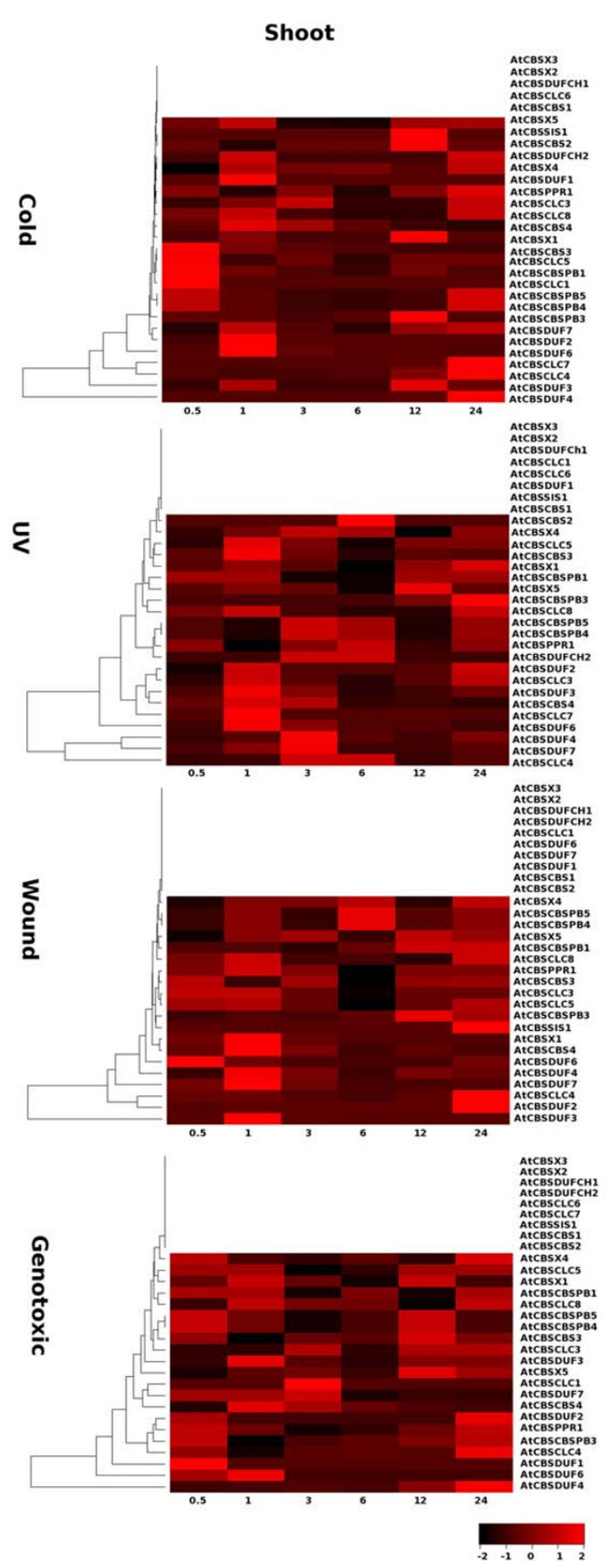

\section{Figure 8}

Heatmap analysis of CDCP genes using microarray data obtained from TAIR 8. The microarray data of the selected gene expression for various abiotic stress conditions such as cold, UV, wound, genotoxic stress were retrieved from TAIR (ver 8). The datasets obtained were corresponding to roots and shoots tissue at different time sets of stress namely $30 \mathrm{~min}, \mathrm{I} \mathrm{h}, 3$ $\mathrm{h}, 6 \mathrm{~h}, 12 \mathrm{~h}$ and $24 \mathrm{~h}$ and analyzed with respect to the control. The empty rows in the heatmap show the unaltered behavior of the respective gene with respect to the control. The hierarchical clustering was performed and heatmaps were generated using gplots package of open source R software respectively. The scale shows the Z-score, which is defined as "actual value" minus the mean of the group divided by the standard deviation. 

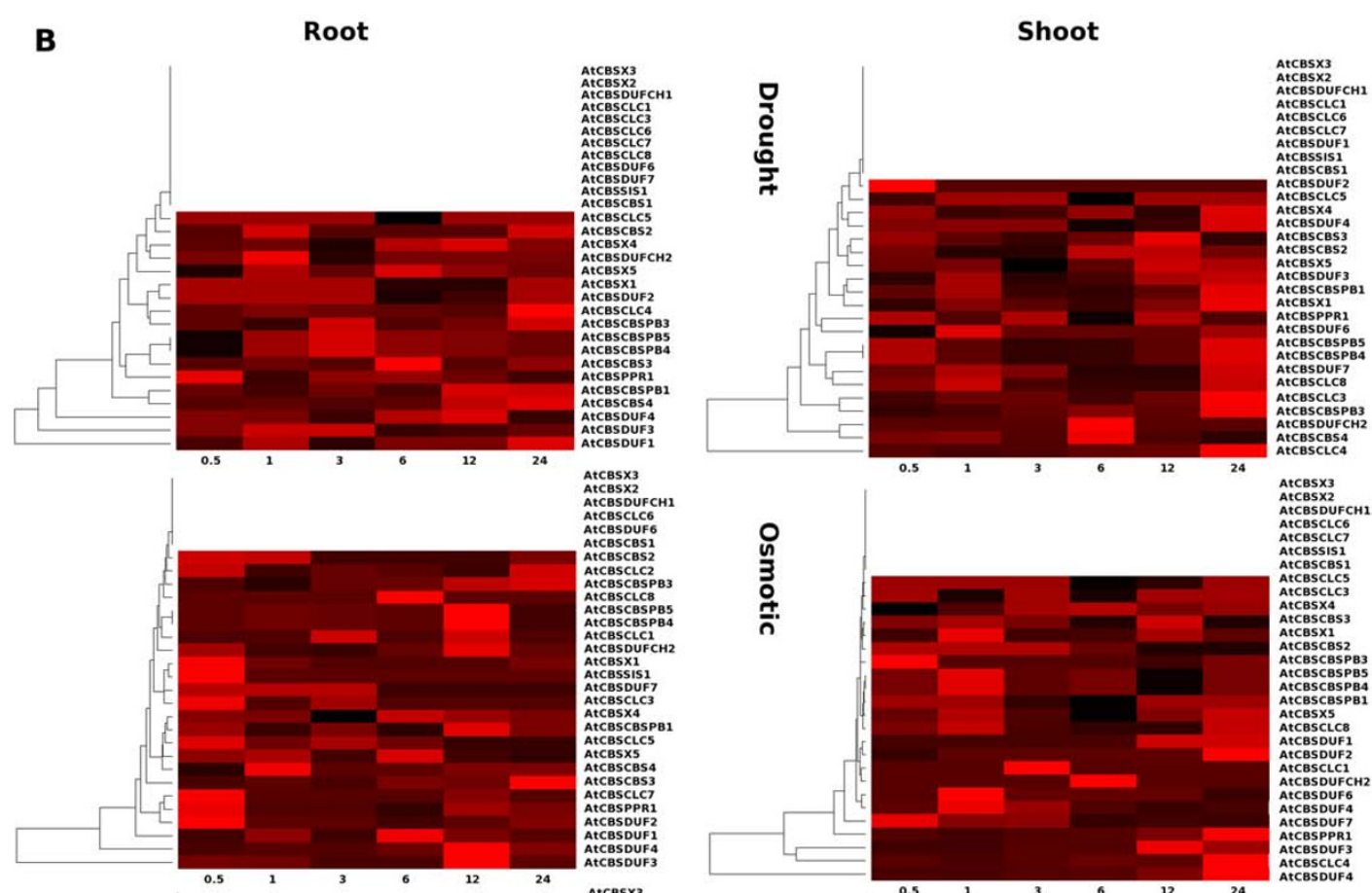

AtCBS $\times 3$
AtCBSX2
AtCBSDUFCH1
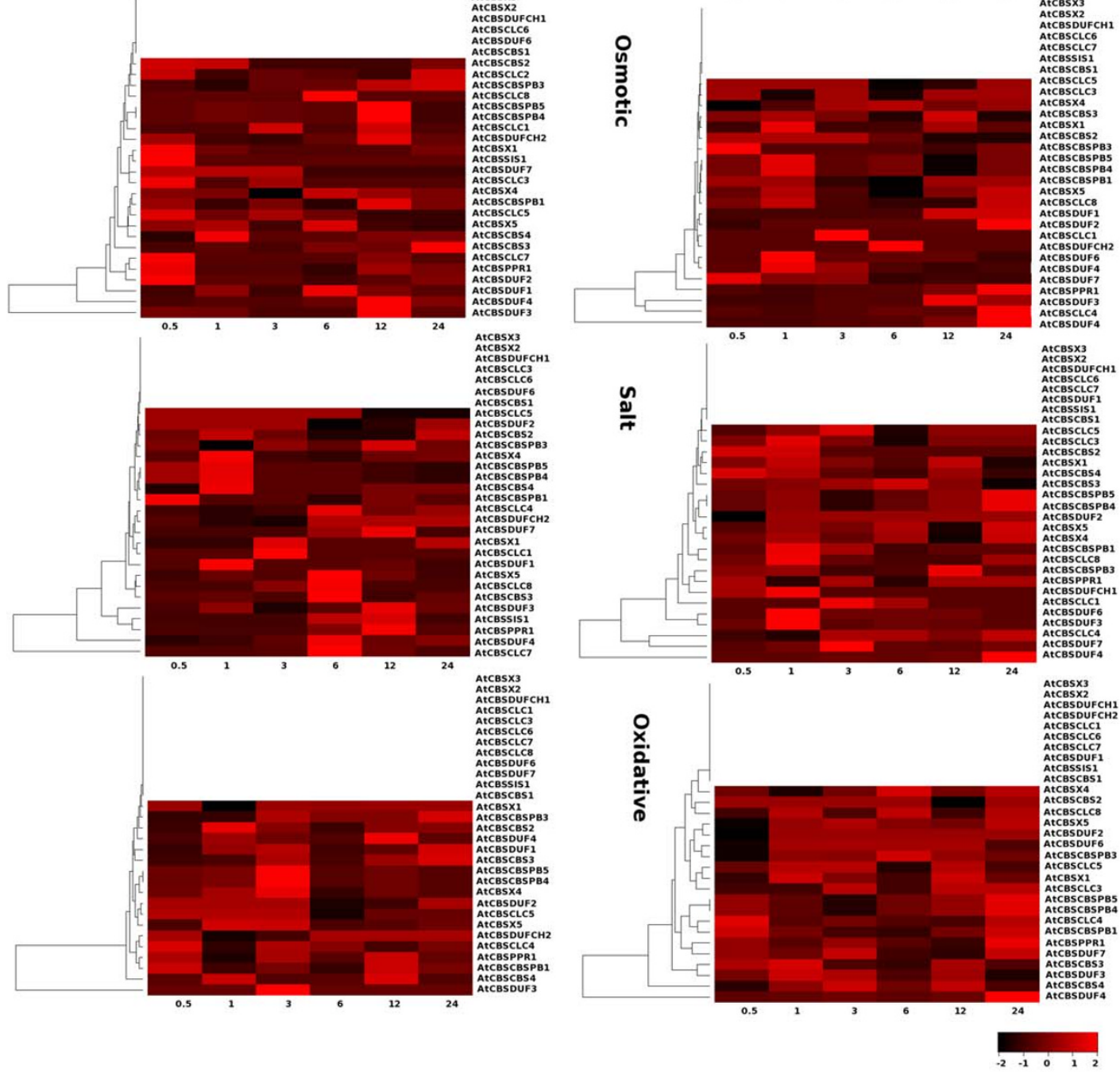

\section{Figure 9}

Heatmap analysis of CDCP genes using microarray data obtained from TAIR 8. The microarray data of the selected gene expression for various abiotic stress conditions such as drought, osmotic, salt and oxidative stress were retrieved from TAIR (ver 8). The datasets obtained were corresponding to roots and shoots tissue at different time sets of stress namely 30 min, I h, $3 \mathrm{~h}, 6 \mathrm{~h}, 12 \mathrm{~h}$ and $24 \mathrm{~h}$ and analyzed with respect to the control. The empty rows in the heatmap show the unaltered behavior of the respective gene with respect to the control. The hierarchical clustering was performed and heatmaps were generated using gplots package of open source R software respectively. The scale shows the Z-score, which is defined as "actual value" minus the mean of the group divided by the standard deviation. 


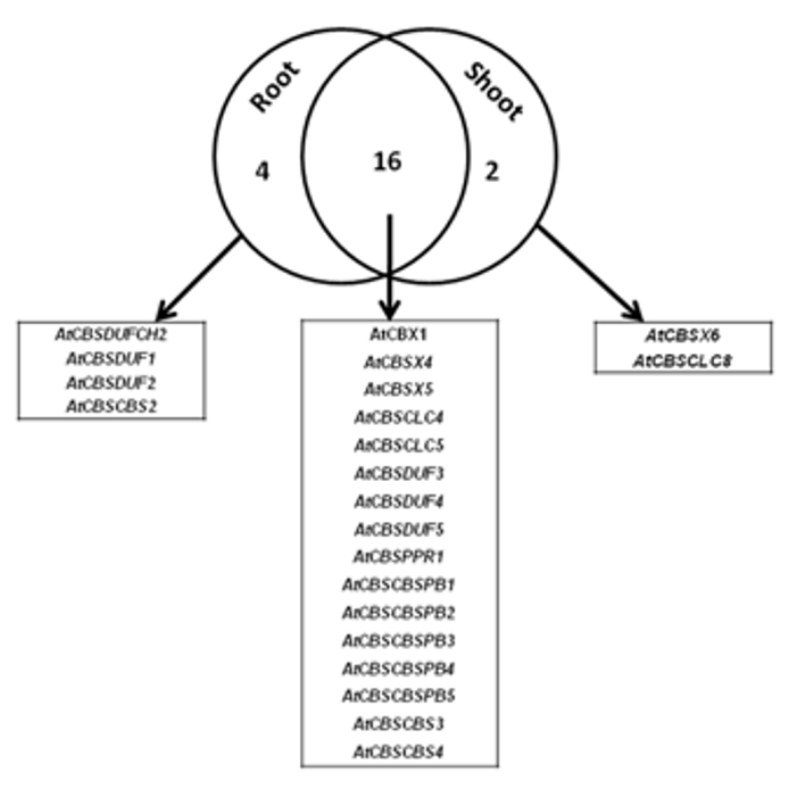

Figure 10

Venn diagram depicting the complexities of tissue specific expression of CDCP genes in root and shoot tissues, under various stress conditions as revealed by microarray expression analysis in Arabidopsis thaliana.

Salt and osmotic stress result in transient increase in a cytoplasmic free calcium concentration, and disruption of this calcium gradient affects downstream gene expression [78]. Earlier, some attempts have been made to identify the osmotic stress related genes in Arabidopsis [79]. Under the condition of osmotic stress, expression of some of the CDCP genes was found to be altered with respect to the control in $30 \mathrm{~min}$ and $12 \mathrm{hr}$ stress in roots, while in shoots these genes showed higher expression at $1 \mathrm{hr}$ and $24 \mathrm{hr}$ of osmotic stress. Under salt stress, CDCP genes were found to be up regulated at 1,6 and $12 \mathrm{hr}$ time points in roots, while in shoots these genes were expressed more in $1 \mathrm{hr}$ and $24 \mathrm{hr}$ of salt stress. The analysis of expression of these genes gives an idea that CBS domain containing proteins might play an important role in drought, salt and osmotic stress response/tolerance. Oxidative stress, arising from an imbalance in the generation and removal of reactive oxygen species (ROS) such as hydrogen peroxide $\left(\mathrm{H}_{2} \mathrm{O}_{2}\right)$, is a challenge faced by all aerobic organisms [80,81]. Although ROS were originally considered to be detrimental to cells, it is now widely recognized that redox regulation involving ROS is a key factor modulating cellular activities [82]. Oxidative stress seems to induce the expression of CDCP genes at $3 \mathrm{hr}$ of stress in roots while in shoot almost all the CDCP genes, which were differentially expressed showed upregulation under oxidative stress.
This analysis revealed two important facts about the CDCP genes. On the one hand, we could identify some members (such as AtCBSX2, AtCBSX3, and AtCBSCBS1; three in total) whose expression was found to be unaltered under any stress conditions, in either roots or shoots. On the other hand, some genes could be identified (such as AtCBSX1 and others shown in Figure 10; sixteen in total) which were found to be differentially expressed with respect to the control under all the stress conditions in both, roots and shoots. Similarly, members such as AtCBSDUFCH2, AtCBSDUF1, AtCBSDUF2 and AtCBSCBS2 were altered under all stresses only in roots (four in total). In contrast, two genes (AtCBSX6 and AtCBSCLC8) could also be identified which were differentially expressed under all stresses only in shoots. It was also observed that more CDCP genes were differentially regulated under all stresses in roots tissues (20 in total) than in shoot tissues (18 in total).

\section{Conclusion}

The cystathionine $\beta$-synthase (CBS) domain containing proteins (CDCPs) comprise of a large superfamily of evolutionarily conserved proteins which are present in all kingdoms of life. In plants, CDCPs were never reported and hence their occurrence and possible function is still a mystery. The present study has identified CDCPs in Arabidopsis thaliana and Oryza sativa at the whole genome level. We also propose their classification based on the presence of CBS domain and various other functional domain(s) present in them. The chromosomal position of genes encoding CDCPs gives an insight into their distribution in the whole genome of Arabidopsis and Oryza. In addition, MPSS analysis reveals the differential expression of these genes in various tissues and parts under stress conditions in these plant species. Moreover, microarray expression data gives an overview that the CDCPs might play an important role in stress response/tolerance in Arabidopsis under various stress conditions. Thus, it can be concluded that CDCP genes exhibit strict stress and developmentally regulated expression patterns in Arabidopsis and Oryza. Certainly, there is a need to functionally validate the role of these CDCPs which exhibit "induced expression patterns" under multiple stresses. Another major finding of this work is the observed expansion of CDCP gene family in Oryza as compared to Arabidopsis. This expansion has been noticed in Oryza primarily because of presence of relatively more cases of alternative splicing as well as gene duplications, possibly indicating towards the significant involvement of CDCPs in development and stress responses. This study will be helpful in commenting on the structural and functional aspects of these unexplored proteins with respect to their roles under various abiotic stresses. Tools of functional genomics based on transgenic approach can further help in testing the "candidature" of these proteins with defined features towards improving 
stress tolerance in crop plants. The leads provided here would also pave the way for elucidating the precise role of individual CDCP proteins in plants.

\section{Methods}

\section{Search and analysis of CBS domain containing proteins} The CDCP protein sequences were retrieved from whole genome sequences of Arabidopsis thaliana (TIGR version 5 and TAIR 8) and Oryza sativa (TIGR version 6). The sequences obtained from TIGR were also cross checked with TAIR8 sequences for any new instances of CDCP proteins in Arabidopsis. The domain structure of the CBS was used to identify and classify the CDCP proteins using the TIGR A. thaliana genome sequence version 5.0 and $O$. sativa version 6.0. Profiles unique to the CBS domain were used to screen all predicted proteins using the HMMER software (version 2.3.2; http://hmmer.wustl.edu/. These unique profiles are for Pfam HMM of CBS domain (accession no. PF00571) [83]. We have used these profiles as default parameters in the hmmsearch program of the HMMER package [84]. All the significant hits having positive scores were selected for classification and were then examined individually for accessory domains that are usually present. This was accomplished by searching the sequences against the Pfam database (version 21.0) to map the known domains, such as Voltage_CLC, CorC_HlyC, SIS, PB1, DUF21 and IMPDH1 (see Fig. 2 for a schematic representation). This step, besides mapping accessory domains to the CBS provides the basis of classifying these proteins. The percentage values of the sequence similarity and identity within the groups were obtained using BLAST. The respective CDCP protein sequences were also cross checked from the TAIR8 database for presence of any additional alternative splice forms in Arabidopsis.

For convenience, we have assigned name to protein sequence according to the domains observed in the respective protein sequence, where At is for Arabidopsis thaliana and Os is for Oryza sativa. The chromosomal positions of the CDCP genes were obtained from TIGR version 6 for $O$. sativa and TIGR version 5, TAIR version 8 for $A$. thaliana, and plotted using Dia version 0.96.1, an open source gtk+ based diagram creation program.

\section{Sequence and phylogenetic analysis}

Multiple alignment analyses were performed using MUSCLE (version 3.6) program [85]. The unrooted parsimonious tree was plotted using protpars and drawtree program of phylip package (version 3.66) [86] using default parameters. Similarity and identity values among the protein sequences were analyzed using standalone BLAST (version 2.2.15) [87]. The figures for final alignment were prepared using Jalview multiple sequence alignment editor [88].

\section{Expression analysis using MPSS database}

Expression evidence from MPSS (Massively Parallel Signature Sequencing) tags was determined from the Arabidopsis MPSS project http://mpss.udel.edu/at/ mapped to Arabidopsis and Oryza gene models. The signature was considered to be significant if it uniquely identifies an individual gene and shows perfect match (100\% identity over 100\% length of the tag). The normalized abundance (tags per million, tpm) of these signatures for a given gene in a given library represents a quantitative estimate of expression of that gene.

The description of MPSS libraries in A. thaliana is: CAF Callus, hardened tissue that forms to protect the exposed areas of cuttings; INF - Inflorescence, part of the plant that consists of flower bearing stalks; LEF - Leaves - 21 day, untreated, classic MPSS; ROF - Root - 21 day, untreated, classic MPSS; SIFSilique (Seedpod) - 24 to 48 hr post-fertilization, classic MPSS; AP1 - ap1-10 inflorescence (part of the plant that consists of flower bearing stalks) - mixed stage, immature buds; AP3 - ap3-6 inflorescence (part of the plant that consists of flower bearing stalks) - mixed stage, immature buds; AGM - agamous inflorescence (part of the plant that consists of flower bearing stalks) mixed stage, immature buds; INS - Inflorescence - mixed stage, immature buds; ROS - Root - 21 day, untreated; SAP - sup/ap1 inflorescence - mixed stage, immature buds; S0 4 - Leaves, 4 hr after salicylic acid treatment; S52 - Leaves, $52 \mathrm{hr}$ after salicylic acid treatment; LES - Leaves - 21 day, untreated; GSE - Germinating seedlings; CAS Callus (hardened tissue that forms to protect the exposed areas of cuttings) - actively growing, signature MPSS; SIS - Silique (Seedpod) - 24 to 48 hr post-fertilization, signature MPSS.

The description of MPSS libraries in O. sativa is: NYR -14 days - Young Roots, NRA - 60 days - Mature Roots - Replicate A, NRB - 60 days - Mature Roots - Replicate B, NGD - 10 days - Germinating seedlings grown in dark, NST - 60 days - Stem, NYL - 14 days - Young leaves, NLA - 60 days - Mature Leaves - Replicate A, NLB - 60 days Mature Leaves - Replicate B, NLC - 60 days - Mature Leaves - Replicate C, NLD - 60 days - Mature Leaves Replicate D, NME - 60 days - Crown vegetative meristematic tissue, NPO - Mature Pollen, NOS - Ovary and mature stigma, NIP - 90 days - Immature panicle, NGS 3 days - Germinating seed, NCA - 35 days - Callus, NSR - 14 days - Young roots stressed in $250 \mathrm{mM} \mathrm{NaCl}$ for 24 h, NSL - 14 days - Young leaves stressed in $250 \mathrm{mM} \mathrm{NaCl}$ for $24 \mathrm{~h}, \mathrm{NDR}$ - 14 days - Young roots stressed in drought for 5 days, NDL - 14 days - Young leaves stressed in drought for 5 days, NCR- 14 days - Young roots stressed in $4 \mathrm{C}$ cold for $24 \mathrm{~h}, \mathrm{NCL}$ - 14 days - Young leaves stressed in $4 \mathrm{C}$ cold for $24 \mathrm{~h}, 9 \mathrm{RO}$ - Roots, I9RR - Roots Replicate, 9LA - Leaves, 9LB - Leaves - Replicate, 9LC - 
Leaves, 9LD - Leaves - Replicate, 9ME - Meristematic Tissue, FRO - F1 Hybrid 60 days Mature Root, FRR - F1 Hybrid 60 days Mature Root-Repl, FLA - F1 Hybrid 60 days Mature Leaf Replicate A, FLB - F1 Hybrid 60 days Mature Leaf Replicate B, FLC - F1 Hybrid 60 days Mature Leaf Replicate C, FLD - F1 Hybrid 60 days Mature Leaf Replicate D, FME - F1 Hybrid 60 days Meristematic tissue, PSC - rice developing seeds, 6 days old cypress high milling (99-1710), PSI - rice developing seeds, 6 days old, Ilpumbyeo - High Taste, PSL - rice developing seeds, 6 days old, LaGrue-Low Milling, PSN - rice developing seed, 6 days old, Nipponbare-Grain quality control, PSY rice developing seeds, 6 days old, Expression values obtained from MPSS database for respective CDCP genes were used for making the heatmap using gplots package of open source R software.

\section{Expression analysis using microarrays}

The microarray data of the selected gene expression for various abiotic stress conditions such as cold, UV, wound, genotoxic stress, drought, osmotic, salt and oxidative stress were retrieved from the Arabidopsis Information Resource [89]. The datasets obtained were corresponding to root and shoot tissues at different time sets of stress namely $30 \mathrm{~min}, 1 \mathrm{hr}, 3 \mathrm{hr}, 6 \mathrm{hr}, 12 \mathrm{hr}$ and $24 \mathrm{hr}$. Fold increase in transcript abundance under stress conditions were calculated with respect to their controls. The transcript abundance with respect to the control was calculated using PERL scripts. The hierarchical clustering analysis and the heatmaps were made using gplots package of R software.

\section{Authors' contributions}

SKS, SLS-P and AP made contributions to the conception of the study and in the preparation of the final draft of the manuscript. HRK, AKS and AP devised overall strategy, performed analysis, drafted and edited the manuscript. HRK developed relevant programs for sequence and microarray analysis. All authors read and approved the final manuscript.

\section{Additional material}

\footnotetext{
Additional file 1

Multiple sequence alignment of amino acid sequence of single CBS domain in Arabidopsis thaliana and Oryza sativa. Multiple sequence alignment of amino acid sequence of single CBS domain in Arabidopsis and Oryza. The alignments of the domain sequences were obtained using MUSCLE software and figures were prepared using multiple sequence alignment editor-Jalview. The consensus sequence is shown below these graphs.

Click here for file

[http://www.biomedcentral.com/content/supplementary/14712164-10-200-S1.jpeg]
}

\section{Additional file 2}

Multiple sequence alignment of amino acid sequence of two CBS domains in Arabidopsis thaliana and Oryza sativa. Multiple sequence alignment of amino acid sequence of two CBS domains in Arabidopsis and Oryza. The alignments of the domain sequences were obtained using MUSCLE software and figures were prepared using multiple sequence alignment editor-Jalview. The consensus sequence is shown below these graphs.

Click here for file

[http://www.biomedcentral.com/content/supplementary/14712164-10-200-S2.jpeg]

\section{Additional file 3}

MPSS analysis of CBS domain containing proteins in Oryza sativa. Expression evidence from MPSS tags was determined from the Oryza sativa MPSS project http://mpss.udel.edu/at/. The normalized abundance (tags per million, tpm) of these signatures for a given gene in a given library represents a quantitative estimate of expression of that gene. The description of these libraries is given in methods.

Click here for file

[http://www.biomedcentral.com/content/supplementary/14712164-10-200-S3.xls]

\section{Additional file 4}

MPSS analysis of CBS domain containing proteins in A. thaliana. Expression evidence from MPSS tags was determined from the Arabidopsis MPSS project http://mpss.udel.edu/at/. The normalized abundance (tags per million, tpm) of these signatures for a given gene in a given library represents a quantitative estimate of expression of that gene. The description of these libraries is given in methods.

Click here for file

[http://www.biomedcentral.com/content/supplementary/14712164-10-200-S4.xls]

\section{Acknowledgements}

Authors would like to thank Jawaharlal Nehru University (JNU), International Center for Genetic Engineering and Biotechnology (ICGEB), Department of Science and Technology and Department of Biotechnology, Govt. of India for financial support. HRK would like to thank Council for Scientific and Industrial Research (CSIR), New Delhi, India for Research Fellowship.

\section{References}

I. Gollery M, Harper J, Cushman J, Mittler T, Girke T, Zhu JK, BaileySerres J, Mittler R: What makes species unique? The contributions of proteins with obscure feature. Genome Biol 2006, 7:757.

2. Gollery M, Harper J, Cushman J, Mittler T, Mittler R: POFs: what we don't know can hurt us. Trends Plant Sci 2007, I 2:492-496.

3. Horan K, Jang C, Bailey-Serres J, Mittler R, Shelton C, Harper JF, Zhu JK, Cushman JC, Gollery M, Girke T: Annotating genes of known and unknown functions by large-scale co-expression analysis. Plant Physiol 2008, 147:4|-57.

4. Luhua S, Ciftci-Yilmaz S, Harper J, Cushman J, Mittler R: Enhanced tolerance to oxidative stress in transgenic Arabidopsis plants expressing proteins of unknown function. Plant Physiol 2008, I 48:280-92.

5. Kumari S, Sabharwal VP, Kushwaha HR, Sopory SK, Singla-Pareek SL, Pareek A: Transcriptome map for seedling stage specific salinity stress response indicates a specific set of genes as candidate for salinetolerance in Oryza sativa L. Func Integr Genomics 2008, 9: 109-123.

6. Ignoul S, Eggermont J: CBS domains: structure, function, and pathology in human proteins. Am J Physiol Cell Physiol 2005, 289:CI369-1378. 
7. Bateman A: The structure of a domain common to archaebacteria and homocystinuria disease proteins. Trends Biochem Sci 1997, 22:12-13.

8. Aitken SM, Kirsch JF: The enzymology of cystathionine biosynthesis: strategies for the control of substrate and reaction specificity. Arch Biochem Biophys 2005, 433: I66-I75.

9. Arad M, Benson DW, Perez-Atayde AR, McKenna WJ, Sparks EA Kanter RJ, McGarry K, Seidman JG, Seidman CE: Constitutively active AMP kinase mutations cause glycogen storage disease mimicking hypertrophic cardiomyopathy. J Clin Invest 2002, I 09:357-362.

10. Blair E, Redwood C, Ashrafian H, Oliveira M, Broxholme J, Kerr B, Salmon A, Ostman-Smith I, Watkins H: Mutations in the $\gamma_{2}$ subunit of AMP-activated protein kinase cause familial hypertrophic cardiomyopathy: evidence for the central role of energy compromise in disease pathogenesis. Hum Mol Genet 200I, 10:1215-1220.

II. Gollob MH, Green MS, Tang AS, Gollob T, Karibe A, Ali Hassan AS, Ahmad F, Lozado R, Shah G, Fananapazir L, et al.: Identification of a gene responsible for familial Wolff-Parkinson-White syndrome. N Engl J Med 200I, 344:|823-|83|

12. Gollob MH, Seger JJ, Gollob TN, Tapscott T, Gonzales O, Bachinski L, Roberts R: Novel PRKAG2 mutation responsible for the genetic syndrome of ventricular preexcitation and conduction system disease with childhood onset and absence of cardiac hypertrophy. Circulation 200I, I 04:3030-3033.

13. Scott JW, Hawley SA, Green KA, Anis M, Stewart G, Scullion GA, Norman DG, Hardie DG: CBS domains form energy-sensing modules whose binding of adenosine ligands is disrupted by disease mutations. J Clin Invest 2004, I I 3:274-284.

14. Bowne SJ, Sullivan LS, Blanton SH, Cepko CL, Blackshaw S, Birch DG Hughbanks-Wheaton D, Heckenlively JR, Daiger SP: Mutations in the inosine monophosphate dehydrogenase I gene (IMPDHI) cause the RPIO form of autosomal dominant retinitis pigmentosa. Hum Mol Genet 2002, I I :559-568.

15. Mahmood NABN, Biemans-Oldehinkel E, Patzlaff JS, Schuurman-Wolters GK, Poolman B: Ion specificity and ionic strength dependence of the osmoregulatory ABC transporter OpuA. J Biol Chem 2006, 28I:29830-29839.

16. Cleiren E, Benichou O, Van Hul E, Gram J, Bollerslev J, Singer FR, Beaverson K, Aledo A, Whyte MP, Yoneyama T, deVernejoul MC, Van Hul W: Albers-Schonberg disease (autosomal dominant osteopetrosis, type II) results from mutations in the CICN7 chloride channel gene. Hum Mol Genet 200I, I 0:286 I-2867.

17. Haug K, Warnstedt M, Alekov AK, Sander T, Ramirez A, Poser B Maljevic S, Hebeisen S, Kubisch C, Rebstock J, et al.: Mutations in CLCN2 encoding a voltage-gated chloride channel are associated with idiopathic generalized epilepsies. Nat Genet 2003 33:527-532

18. Konrad M, Vollmer M, Lemmink HH, Heuvel LP van den, Jeck N, Vargas-Poussou R, Lakings A, Ruf R, Deschenes G, Antignac C, et al. Mutations in the chloride channel gene CLCNKB as a cause of classic Bartter syndrome. I Am Soc Nephrol 2000 I I:|449-|459.

19. Kornak U, Kasper D, Bosl MR, Kaiser E, Schweizer M, Schulz A, Friedrich W, Delling G, Jentsch T]: Loss of the CIC-7 chloride channel leads to osteopetrosis in mice and man. Cell 200I, 1 04:205-215.

20. Lloyd SE, Gunther W, Pearce SH, Thomson A, Bianchi ML, Bosio M Craig IW, Fisher SE, Scheinman SJ, Wrong O, Jentsch TJ, Thakker RV: Characterisation of renal chloride channel, CLCN5, mutations in hypercalciuric nephrolithiasis (kidney stones) disorders. Hum Mol Genet 1997, 6:1233-1239.

21. Pusch M: Myotonia caused by mutations in the muscle chloride channel gene CLCN I. Hum Mutat 2002, 19:423-434.

22. Lurin C, Güclü J, Cheniclet C, Carde JP, Barbier-Brygoo H, Maurel C CLC-NtI, a putative chloride channel protein of tobacco, colocalizes with mitochondrial membrane markers. Biochem J 2000, 348:291-295.

23. Hechenberger M, Schwappach B, Fischer WN, Frommer WB, Jentsch T], Steinmeyer K: A family of putative chloride channels from Arabidopsis and functional complementation of a yeast strain with aCLC gene disruption. I Biol Chem 1996 271:33632-33638

24. Geelen D, Lurin C, Bouchez D, Frachisse JM, Lelievre F, Courtial B, Barbier-Brygoo H, Maurel C: Disruption of putative anion chan- nel gene AtCLC-a in Arabidopsis suggests a role in the regulation of nitrate content. Plant / 2000, 2 I:259-267.

25. Marmagne A, Vinauger-Douard $M$, Monachello D, Longevialle AF, Charon C, Allot M, Rappaport F, Wollman FA, Barbier-Brygoo H, Ephritikhine G: Two members of the Arabidopsis CLC (chloride channel) family, AtCLCe and AtCLCf, are associated with thylakoid and Golgi membranes, respectively. J Exp Bot 2007, 58:3385-3393.

26. Nakamura A, Fukuda A, Sakai S, Tanaka Y: Molecular cloning, functional expression and subcellular localization of two putative vacuolar voltage-gated chloride channels in Rice (Oryza sativa L.). Plant Cell Physiol 2006, 47:32-42.

27. Arabidopsis Genome Initiative: Analysis of the genome sequence of the flowering plant Arabidopsis thaliana. Nature 2000, 408:796-8I5.

28. Pareek A, Singh A, Kumar M, Kushwaha HR, Lynn AM, Singla-Pareek SL: Whole genome analysis of Oryza sativa $L$. reveals similar architecture of two-component-signaling-machinery with Arabidopsis. Plant Physiol 2006, I 42:380-397.

29. Gibson MM, Bagga DA, Miller CG, Maguire ME: Magnesium transport in Salmonella typhimurium : the influence of new mutations conferring $\mathrm{Co}^{2+}$ resistance on the CorA $\mathrm{Mg}^{2+}$ transport system. Mol Microbiol I991, 5:2753-2762.

30. ter Huurne AA, Muir S, van Houten M, Zeijst BA van der, Gaastra W, Kusters JG: Characterization of three putative Serpulina hyodysenteriae hemolysins. Micro Patho 1994, 16:269-282

31. Mindell JA, Maduke M: CIC chloride channels. Genome Biol 200I, 2:1-3003

32. Zhang J, George AL Jr, Griggs RC, Fouad GT, Roberts J, Kwiecinski $\mathrm{H}$, Connolly AM, Ptacek LJ: Mutations in the human skeletal muscle chloride channel gene (CLCNI) associated with dominant and recessive myotonia congenita. Neurology 1996, 47:993-998

33. Schmidt-Rose T, Jentsch T): Reconstitution of functional voltage-gated chloride channels from complementary fragments of CLC-I. J Biol Chem 1997, 272:205 I5-2052I.

34. Bateman A: The SIS domain: a phosphosugar-binding domain. Trends Biochem Sci 1999, 24:94-95.

35. Teplyakov A, Obmolova G, Badet-Denisot MA, Badet B, Polikarpov I: Involvement of the $\mathbf{C}$ terminus in intramolecular nitrogen channeling in glucosamine 6-phosphate synthase: evidence from a I.6 A crystal structure of the isomerase domain. Structure 1998, 6: 1047-1055.

36. Small ID, Peeters I: The PPR motif - a TPR-related motif prevalent in plant organellar proteins. Trends Biochem Sci 2000, 25:45-47.

37. Barkan A, Walker M, Nolasco M, Johnson D: A nuclear mutation in maize blocks the processing and translation of several chloroplast mRNAs and provides evidence for the differential translation of alternative mRNA forms. EMBO J 1994 , I3:3170-3 I8I.

38. Manthey GM, McEwen JE: The product of the nuclear genePET309 is required for translation of mature mRNA and stability or production of intron-containing RNAs derived from the mitochondrial COXI locus of Saccharomyces cerevisiae. EMBO J 1995, 1 4:403 I-4043.

39. English JM, Vanderbilt CA: Isolation of MEK5 and differential expression of alternatively spliced forms. J Biol Chem 1995 270:28897-28902

40. Zhou G, Bao ZQ, Dixon JE: Components of a new human protein kinase signal transduction pathway. J Biol Chem 1995, 270: $12665-12669$.

41. Ponting CP: Novel domains in NADPH oxidase subunits, sorting nexins, and Ptdlns 3-kinases: binding partners of $\mathrm{SH}_{3}$ domains? Protein Sci 1996, 5:2353-2357.

42. Campbell MA, Haas BJ, Hamilton JP, Mount SM, Buell CR: Comprehensive analysis of alternative splicing in rice and comparative analyses with Arabidopsis. BMC Genomics 2006, 7:327.

43. Sintchak MD, Fleming MA, Futer O, Raybuck SA, Chambers SP, Caron $P R$, et al: Structure and mechanism of inosine monophosphate dehydrogenase in complex with the immunosuppressent mycophenolic acid. Cell 1996, 85:921-930.

44. Hardie DG, Hawley SA: AMP-activated protein kinase: the energy change hypothesis revisited. Bioessay 200I, 23: $1112-1119$. 
45. Jeong BC, Yoo KS, Jung KW, Shin JS, Song HK: Purification, crystallization and preliminary $X$-ray diffraction analysis of a cystathionine b-synthase domain-containing protein, CDCP2 from Arabidopsis thaliana. Acta Crystallographica 2008, F64:825-827.

46. Estévez R, Pusch M, Ferrer-Costa C, Orozco M, Jentsch TJ: Functional and structural characteristic conservation of BCS domains from CLC chloride channels. J Physiol 2004, 557:363-378.

47. Schwappach B, Stobrawa S, Hechenberger M, Steinmeyer K, Jentsch TJ: Golgi localization and functionally important domain in the $\mathrm{NH} 2$ and $\mathrm{COOH}$ terminus of the yeast CLC putative chloride channels Geflp. J Biol Chem 1998, 273:I5II0-15II8.

48. Dhedhiou CJ, Golldack D: Salt-dependent regulation of chloride channel transcripts in rice. Plant Science 2006, 170:793-800.

49. Ouyang $Z$, Isaacson R: Identification of a novel regulatorfor the Escherichia coli fit iron transport system. Open Microbio J 2008, 2:94-99.

50. Wise RP, Pring DR: Nuclear-mediated mitochondrial gene regulation and male fertility in higher plants: Light at the end of the tunnel? Proc Natl Acad Sci 2002, 99: 10240-10242.

5I. Lurin $C$, Andrés $C$, Aubourg $S$, Bellaoui $M$, Bitton $F$, Bruyère $C$, Caboche M, Debast C, Gualberto J, Hoffmann B, Lecharny A, Le Ret M, Martin-Magniette ML, Mireau H, Peeters N, Renou JP, Szurek B, Taconnat L, Small I: Genome-wide analysis of Arabidopsis pentatricopeptide repeat proteins reveals their essential role in organelle biogenesis. Plant Cell 2004, 16:2089-2 I03.

52. Saha D, Prasad AM, Srinivasan R: Pentatricopeptide repeat proteins and their emerging roles in plants. Plant Physiol Biochem 2007, 45:52I-534.

53. Moscat J, Diaz-Meco MT, Albert A, Campuzano S: Cell signaling and function organized by PBI domain interactions. Mol Cell 2006, 23:63I-640.

54. Sumimoto $\mathrm{H}$, Kamakura $\mathrm{S}$, Ito $\mathrm{T}$ : Structure and function of the PBI domain, a protein interaction module conserved in animals, fungi, amoebas, and plants. SCi STKE 2007, 40 I :re6.

55. Jain M, Tyagi AK, Khurana JP: Genome-wide analysis, evolutionary expansion, and expression of early auxin-responsive SAUR gene family in rice (Oryza sativa). Genomics 2006, 88:360-37I.

56. Nijhawan A, Jain M, Tyagi AK, Khurana JP: Genomic survey and gene expression analysis of the basic leucine zipper transcription factor family in rice. Plant Physiol 2008, 146:333-350

57. Blanc G, Barakat A, Guyot R, Cooke R, Delseny M: Extensive duplication and reshuffling in the Arabidopsis genome. Plant $\mathrm{Cell}$ 2000, I 2:1093-II01.

58. Simillion C, Vandepoele K, Van Montagu MC, Zabeau M, Peer Y Van de: The hidden duplication past of Arabidopsis thaliana. Proc Natl Acad Sci 2002, 99:। 3627-I3632.

59. Vision TJ, Brown DG, Tanksley SD: The origins of genomic duplications in Arabidopsis. Science 2000, 290:2 II4-2117.

60. Blanc G, Hokamp K, Wolfe $\mathrm{KH}$ : A recent polyploidy superimposed on older large-scale duplications in the Arabidopsis genome. Genome Res 2003, 13:137-144.

61. Wang X, Shi X, Hao B, Ge S, Luo J: Duplication and DNA segmental loss in the rice genome: implications for diploidization. New Phytol 2005, 165:937-946.

62. Ermolaeva MD, Wu MM, Eisen JA, Salzberg SL: The age of the Arabidopsis thaliana genome duplication. Plant Mol Biol 2003, 5 I:859-866.

63. Bowers JE, Chapman BA, Rong J, Paterson AH: Unravelling angiosperm genome evolution by phylogenetic analysis of chromosomal duplication events. Nature 2003, 422:433-438.

64. Zhang L, Vision T], Gaut BS: Patterns of nucleotidesubstitution among simultaneously duplicated gene pairs in Arabidopsis thaliana. Mol Biol Evol 2002, 19:1464-I473.

65. Ziolkowski PA, Blanc G, Sadowski J: Structural divergence of chromosomal segments that arose from successive duplication events in the Arabidopsis genome. Nucleic Acids Res 2003 3I:I339-1350

66. Brenner S, Johnson M, Bridgham J, Golda G, Lloyd DH, Johnson D, Luo S, McCurdy S, Foy M, Ewan M, et al.: Gene expression analysis by massively parallel signature sequencing (MPSS) on microbead arrays. Nat Biotechnol 2000, 18:630-634.

67. Thomashow MF: Arabidopsis thaliana as a model for studying mechanisms of plant cold tolerance. In Arabidopsis Edited by:
Meyerowitz E, Somerville C. Cold Spring Harbor Laboratory Press, Cold Spring Harbor, NY; 1994:807-834.

68. Kimura M, Yoshizumi T, Manabe K, Yamamoto YY, Matsui M: Arabidopsis transcriptional regulation by light stress via hydrogen peroxide-dependent and -independent pathways. Genes Cells 200I, 6:607-6I7.

69. Reymond P, Weber H, Damond M, Farmer EE: Differential gene expression in response to mechanical wounding and insect feeding in Arabidopsis. Plant Cell 2000, 12:707-719.

70. Heribert Hirt, Kazuo Shinozaki: Plant Responses to Abiotic Stress. In Topics in Current Genetics Volume 4. Springer; 2004.

7I. Vieira da Silva J, Naylor AW, Kramer J: Some ultrastructural and enzymatic effects of water stress in cotton (Gossypium L.) leaves. Proc Natl Acad Sci 1974, 71:3243-3247.

72. Pham-Thi AT, Borrel-Flood C, Vieira da Silva J, Justin AM, Mazliak P. Effects of drought on [I-I4C]-oleic and [I-I4C]-linoleic acid desaturation in cotton leaves. Physiol Plant 1987, 69: I47-I50.

73. Monteiro de Paula F, Pham Thi AT, Vieira dailva J, Justin AM, Demandre C, Mazliak P: Effects of water stress on the molecular species composition of polar lipids from Vigna unguiculata $\mathrm{L}$. leaves. Plant Sci 1990, 66:185-193.

74. Ferrari-lliou R, d'Arcy-Lameta A, Pham-Thi AT, Zuily-Fodil Y, Mazliak $P$ : Effect of drought on photodynamic peroxidation of leaf total lipophilic extracts. Phytochem 1994, 37: I237-1243.

75. Sahsah Y, Campos P, Gareil M, Zuily-Fodil Y, Pham-Thi AT: Enzymatic degradation of polar lipids in Vigna unguiculata leaves and influence of drought stress. Physiol Plant 1998, 104:577-586.

76. El Maarouf H, Zuily-Fodil Y, Gareil M, d'Arcy-Lameta A, Pham-Thi AT: Enzymatic activity and gene expression under water stress of phospholipase $D$ in two cultivars of Vigna unguiculata (L.). Plant Mol Biol 1999, 39: 1257-1265.

77. Matos AR, d'Arcy-Lameta A, França M, Petres S, Edelman L, Kader J, Zuily-Fodil Y, Pham-Thi AT: A novel patatin-like gene stimulated by drought stress encodes a galactolipid acyl hydrolase. FEBS Lett 200I, 491: I88-192.

78. Knight $H$, Trewavas AJ, Knight MR: Calcium signalling in Arabidopsis thaliana responding to drought and salinity. Plant $J$ 1997, I 2:1067-1078.

79. Xiong L, Lee H, Ishitani M, Zhu JK: Regulation of Osmotic Stressresponsive Gene Expression by the LOS6/ABAI Locus in Arabidopsis. J Biol Chem 2002, 277:8588-8596.

80. Finkel T, Holbrook NJ: Oxidants, oxidative stress and the biology of ageing. Nature 2000, 408:239-247.

81. Allen RG, Tresini M: Oxidative stress and gene regulation. Free Radic Biol Med 2000, 28:463-499.

82. Dat JF, Lopez-Delgado H, Foyer $\mathrm{CH}$, Scott IM: Parallel changes in $\mathrm{H} 2 \mathrm{O} 2$ and catalase during thermotolerance induced by salicylic acid or heat acclimation in mustard seedlings. Plant Physiol 1998, I I 6: I35I-1357.

83. Finn RD, Mistry J, Schuster-Bockler B, Griffiths-Jones S, Hollich V, Lassmann T, Moxon S, Marshall M, Khanna A, Durbin R, Eddy SR, Sonnhammer EL, Bateman A: Pfam: clans, web tools and services. Nucl Acids Res 2006, 34:247-25I.

84. HMMER [http://hmmer.janelia.org]

85. Edgar, Robert C: MUSCLE: multiple sequence alignment with high accuracy and high throughput. Nucleic Acids Res 2004, 32: 1792-1797.

86. Felsenstein J: PHYLIP (Phylogeny Inference Package). In version 3.6. Distributed by the author Department of Genome Sciences, University of Washington, Seattle; 2005

87. Altschul SF, Gish W, Miller W, Myers EW, Lipman DJ: Basic local alignment search tool. J Mol Biol 1990, 215:403-4I0.

88. Clamp M, Cuff J, Searle SM, Barton GJ: The Jalview Java Alignment Editor. Bioinformatics 2004, 20:426-427.

89. Kilian J, Whitehead D, Horak J, Wanke D, Weinl S, Batistic O, D'Angelo Bornberg-Bauer E, Kudla J, Harter K: The AtGenExpress global stress expression data set: protocols, evaluation and model data analysis of UV-B light, drought and cold stress responses. Plant J 2007, 50:347-363. 